This is a preprint of: "The 16th Hilbert problem for discontinuous piecewise isochronous centers of degree one or two separated by a straight line", Marina Esteban, Jaume Llibre, Clàudia Valls, Chaos, vol. 31, 043112:1-18, 2021.

DOI: $[10.1063 / 5.0023055]$

\title{
The 16th Hilbert problem for discontinuous piecewise isochronous centers of degree one or two separated by a straight line
}

M. Esteban, ${ }^{\text {a) }}$ J. Llibre, ${ }^{\text {b) }}$ and C. Valls ${ }^{\mathrm{c})}$

(Dated: March 7, 2021)

In this paper we deal with discontinuous piecewise differential systems formed by two differential systems separated by a straight line when these two differential systems are linear centers (which always are isochronous) or quadratic isochronous centers. It is known that there is a unique family of linear isochronous centers and four families of quadratic isochronous centers. Combining these five types of isochronous centers we obtain fifteen classes of discontinuous piecewise differential systems.

We provide upper bounds for the maximum number of limit cycles that these fifteen classes of discontinuous piecewise differential systems can exhibit, so we have solved the 16th Hilbert problem for such differential systems. Moreover in seven of the classes of these discontinuous piecewise differential systems the obtained upper bound on the maximum number of limit cycles is reached.

PACS numbers: $02.30 . \mathrm{Hq}$

\footnotetext{
a) marinaep@us.es; Dept. Matemática Aplicada II and Instituto de Matemáticas (IMUS),

Escuela Técnica Superior de Ingeniería de la Universidad de Sevilla,

Camino de los Descubrimientos s/n, 41092 Sevilla (SPAIN)

b)jllibre@mat.uab.cat; Dept. Matemàtiques, Universitat Autònoma de Barcelona, 08193 Bellaterra, Barcelona, Catalonia (SPAIN)

c)cvalls@math.ist.utl.pt; Dept. Matemática, Instituto Superior Técnico, Universidade Técnica de Lisboa, Av. Rovisco Pais 1049-001, Lisboa (PORTUGAL)
} 
To solve the 16th Hilbert problem, i.e. to find an upper bound for the maximum number of limit cycles that a given class of differential systems can exhibit, is in general an unsolved problem. For the classes of discontinuous piecewise differential systems here studied we can obtain the solution using the first integrals of the linear and quadratic isochronous centers.

\section{INTRODUCTION AND MAIN RESULTS}

We consider planar differential systems of the form

$$
\frac{d x}{d t}=P(x, y), \quad \frac{d y}{d t}=Q(x, y)
$$

where $P(x, y)$ and $Q(x, y)$ are polynomial functions, and the degree of the systems is the maximum degree of such polynomials. In particular in this paper we consider discontinuous piecewise differential systems of the form

$$
(\dot{x}, \dot{y})=\mathbf{F}(x, y)=\left\{\begin{array}{l}
\mathbf{F}^{-}(x, y)=\left(f^{-}(x, y), g^{-}(x, y)\right) \text { if } x<0 \\
\mathbf{F}^{+}(x, y)=\left(f^{+}(x, y), g^{+}(x, y)\right) \text { if } x>0
\end{array}\right.
$$

being bi-valued on the separation line $x=0$. Following ${ }^{9}$ a point $(0, y)$ is a crossing point if $f^{-}(0, y) f^{+}(0, y)>0$. If there exists a periodic orbit of the discontinuous differential system (1) having exactly two crossing points, then we call it a crossing periodic orbit. A crossing limit cycle is an isolated periodic orbit in the set of all crossing periodic orbits of system (1). In what follows for simplicity we shall say limit cycle instead of crossing limit cycle.

The analysis of planar continuous piecewise linear systems is well established when the number of linear zones is small, see ${ }^{33}$ and the references therein. They frequently appear in many non-linear engineering devices, which are accurately modelled by piecewise linear vector fields, see ${ }^{7}$. They appear also in mathematical biology, see ${ }^{6,30-32}$. However when the planar vector field is discontinuous, the adaptation of the 16th Hilbert's problem on the maximum number of existing limit cycles, is an open problem. In the last years 
many authors have worked in this problem, trying to determine how many limit cycles can appear in planar systems separated by a straight line, see for instance ${ }^{1-4,8,10-14,16-18,21-29}$. For details on the classical 16th Hilbert problem see for instance ${ }^{15,19,20}$.

Let $p \in \mathbb{R}^{2}$ be a singularity of a differential system in the plane. The singularity $p$ is a center if there exists an open neighbourhood $U$ of $p$ such that all the solutions in $U \backslash\{p\}$ are periodic. Denote by $T_{q}$ the period of the periodic orbit through $q \in U \backslash\{p\}$. We say that $p$ is an isochronous center if $T_{q}$ is constant for all $q \in U \backslash\{p\}$.

In this paper we work with the following five types of systems which cover the classes of a linear system having a center and of all quadratic polynomial differential systems having an isochronous center. For a proof of the linear system see Lemma 1 of $^{27}$ and for a proof of the quadratic systems see page 34 of $^{5}$.

(I) Any linear differential system having a center can be written as

$$
\dot{x}=-A x-\left(A^{2}+\omega^{2}\right) y+B, \quad \dot{y}=x+A y+C,
$$

with $\omega>0, A, B, C \in \mathbb{R}$ and $A \neq 0$. A first integral of this system is

$$
H_{1}(x, y)=(x+A y)^{2}+2(C x-B y)+y^{2} \omega^{2} .
$$

Of course every linear center is isochronous.

(II) The first family of quadratic isochronous differential systems can be obtained doing an affine transformation to the system

$$
\dot{x}=-y+x^{2}-y^{2}, \quad \dot{y}=x(1+2 y),
$$

with first integral

$$
\tilde{H}_{2}(x, y)=\frac{x^{2}+y^{2}}{1+2 y} .
$$

(III) The second family of quadratic isochronous differential systems can be obtained 
doing an affine transformation to the system

$$
\dot{x}=-y+x^{2}, \quad \dot{y}=x(1+y)
$$

whose first integral is

$$
\tilde{H}_{3}(x, y)=\frac{x^{2}+y^{2}}{(1+y)^{2}}
$$

(IV) The third family of quadratic isochronous differential systems can be obtained doing an affine transformation to the system

$$
\dot{x}=-y+\frac{4}{3} x^{2}, \quad \dot{y}=x\left(1-\frac{16}{3} y\right)
$$

with first integral

$$
\tilde{H}_{4}(x, y)=\frac{9\left(x^{2}+y^{2}\right)-24 x^{2} y+16 x^{4}}{-3+16 y}
$$

(V) The fourth family of quadratic isochronous differential systems can be obtained doing an affine transformation to the system

$$
\dot{x}=-y+\frac{16}{3} x^{2}-\frac{4}{3} y^{2}, \quad \dot{y}=x\left(1+\frac{8}{3} y\right)
$$

whose first integral is

$$
\tilde{H}_{5}(x, y)=\frac{9\left(x^{2}+y^{2}\right)+24 y^{3}+16 y^{4}}{(3+8 y)^{4}}
$$

Our objective is to solve the 16th Hilbert problem for the 15 classes of discontinuous piecewise differential systems separated by a straight line and formed by two arbitrary isochronous centers of degree 1 or 2, i.e. we shall provide for all these 15 classes an upper bound on the maximum number of limit cycles that each class can exhibit. Moreover as we shall see in many cases the upper bound that we shall provide is reached.

We must mention that, in general it is very difficult (many times for the moment 
impossible) to provide an upper bound for the maximum number of limit cycles that a class of differential systems in the plane can exhibit, and of course it is even more difficult to provide the exact upper bound, see for instance ${ }^{15,19,20}$.

It was proved in Theorem 3 of $^{27}$, or in Corollary 3 of $^{23}$ that discontinuous piecewise differential systems separated by a straight line and formed by two arbitrary linear centers has no limit cycles. So this case is not considered here.

Our first main result is to provide the maximum number of limit cycles that can exist for discontinuous piecewise differential systems of the form (1), where in $x<0$ there is an arbitrary linear differential center (I), and for $x>0$ there is one of the four quadratic isochronous differential systems (II), (III), (IV) or (V), after an arbitrary affine change of variables.

Theorem 1. Consider discontinuous piecewise differential systems separated by the straight line $x=0$ and formed by a linear differential center (I) after an affine change of variables in $x<0$, and by a quadratic isochronous system of type either (II), or (III), or $(I V)$, or $(V)$ after an affine change of variables in $x>0$. The maximum number of limit cycles of these discontinuous piecewise differential systems are

(a) at most one for systems of type (I)-(II), and there are systems of this type with exactly one limit cycle, see Figure 1;

(b) at most one for systems of type (I)-(III), and there are systems of this type with exactly one limit cycle, see Figure 2;

(c) at most two for systems of type (I)-(IV), and there are systems of this type with exactly one limit cycle, see Figure 3;

(d) at most two for systems of type (I)-(V), and there are systems of this type with exactly two limit cycles, see Figure 4.

Note that for all systems of type (I)-(k) with $k \in\{I I, I I I, V\}$ the upper bound on the maximum number of limit cycles is reached.

The proof of Theorem 1 is given in section III. 


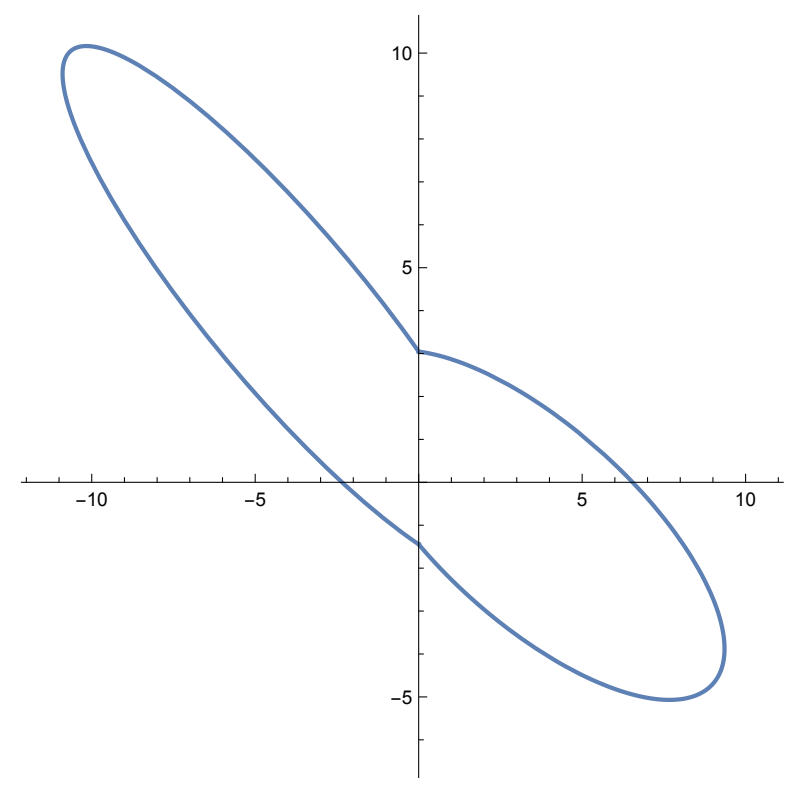

Figure 1: The unique limit cycle that exists for system (8)-(9) of class (I)-(II). It is travelled in counter-clockwise sense.

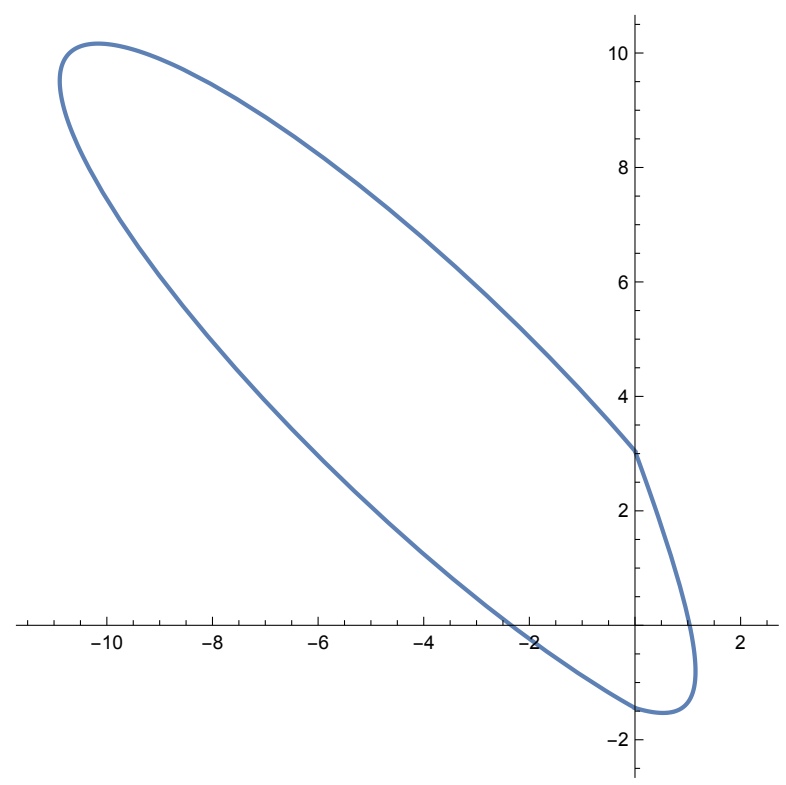

Figure 2: The unique limit cycle that exists for system (8)-(11) of class (I)-(III). It is travelled in counter-clockwise sense.

The second main result of the paper is to give the maximum number of limit cycles that can appear in discontinuous piecewise differential systems of the form (1) such that in a half-plane there is a general quadratic isochronous differential system of type (II), 


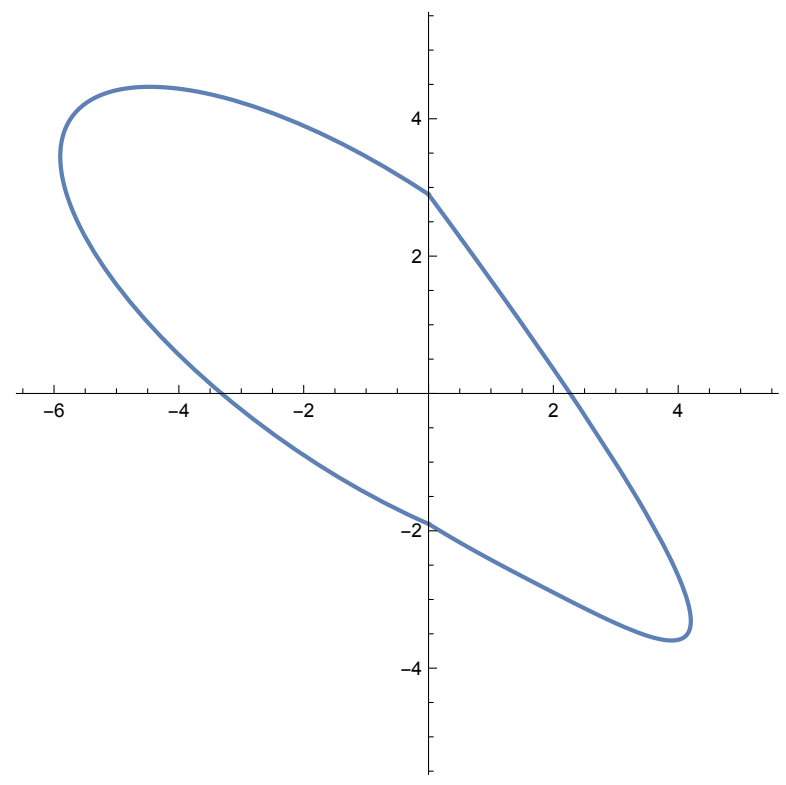

Figure 3: The existing limit cycle for system (13)-(14) of class (I)-(IV). It is travelled in counter-clockwise sense.
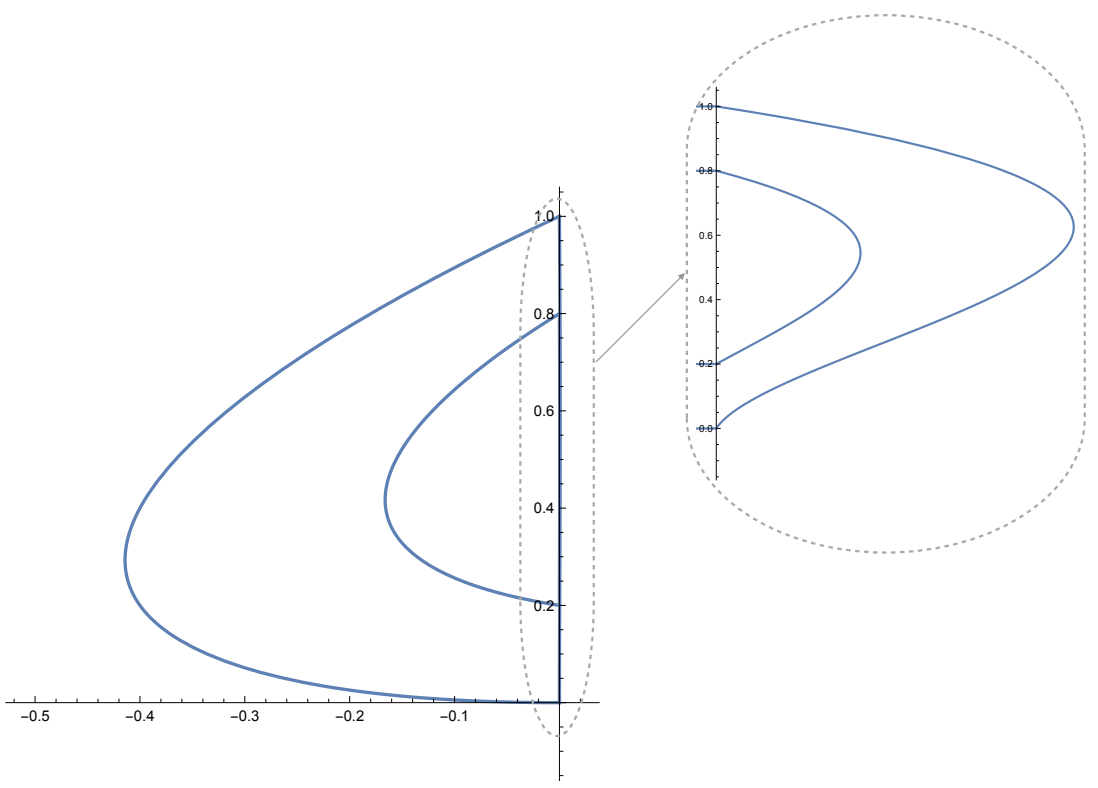

Figure 4: The pair of limit cycles that exist for system (16)-(17) of class (I)-(V). They are travelled in counter-clockwise sense.

and in the other one there is a general quadratic isochronous differential system of type 
(II), (III), (IV) or (V) after an arbitrary affine change of variables.

Theorem 2. Consider discontinuous piecewise differential systems separated by the straight line $x=0$ and formed by a quadratic isochronous center of type (II) after an affine change of variables in $x<0$, and by a quadratic isochronous system of type either $(I I)$, or (III), or (IV), or (V) after an affine change of variables in $x>0$. The maximum number of limit cycles of these discontinuous piecewise differential systems are

(a) at most one for systems of type (II)-(II), and there are systems of this type with exactly one limit cycle, see Figure 5;

(b) at most one for systems of type (II)-(III), and there are systems of this type with exactly one limit cycle, see Figure 6;

(c) at most three for systems of type (II)-(IV), and there are systems of this type with exactly two limit cycles, see Figure 7;

(d) at most three for systems of type (II)-(V), and there are systems of this type with exactly two limit cycles, see Figure 8.

Note that for systems of type (II)-(II) and (II)-(III) after an affine change of variables the upper bound on the maximum number of limit cycles is reached.

The proof of Theorem 2 is given in section IV.

The third main result of the paper is to give the maximum number of limit cycles that can appear in discontinuous piecewise differential systems of the form (1) such that in a half-plane there is a general quadratic isochronous differential system of type (III), and in the other one a general quadratic isochronous differential system of type (III), (IV) or (V) after an arbitrary affine change of variables.

Theorem 3. Consider discontinuous piecewise differential systems separated by the straight line $x=0$ and formed by a quadratic isochronous center of type (III) after an affine change of variables in $x<0$, and by a quadratic isochronous system of type either (III), or (IV), or (V) after an affine change of variables in $x>0$. The maximum number of limit cycles of these discontinuous piecewise differential systems are 


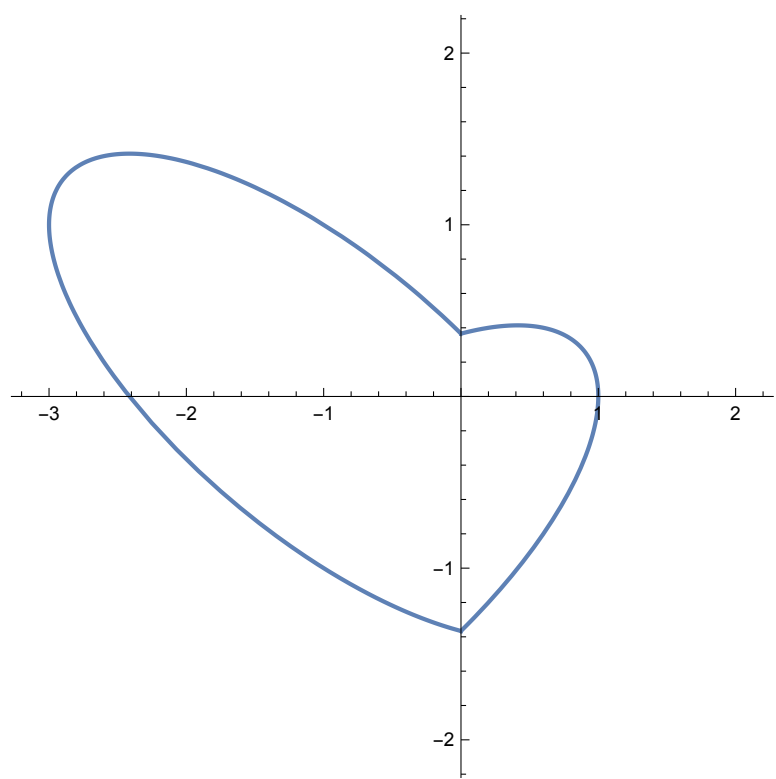

Figure 5: The unique limit cycle that exists for system (20)-(21) of type (II)-(II). It is travelled in counter-clockwise sense.

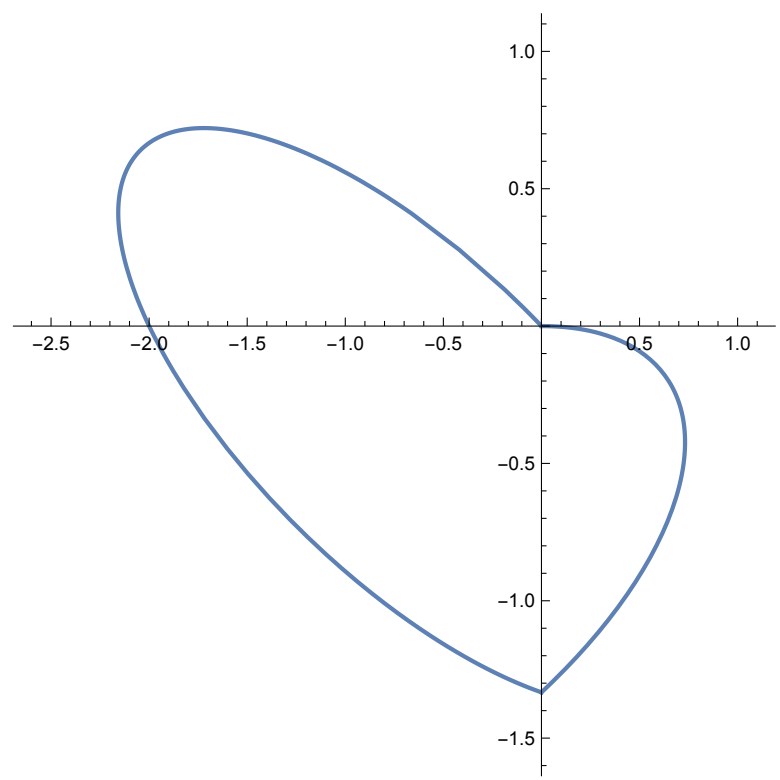

Figure 6: The unique limit cycle that exists for system (20)-(23) of type (II)-(III). It is travelled in counter-clockwise sense.

(a) at most one for systems of type (III)-(III), and there are systems of this type with exactly one limit cycle, see Figure 9; 


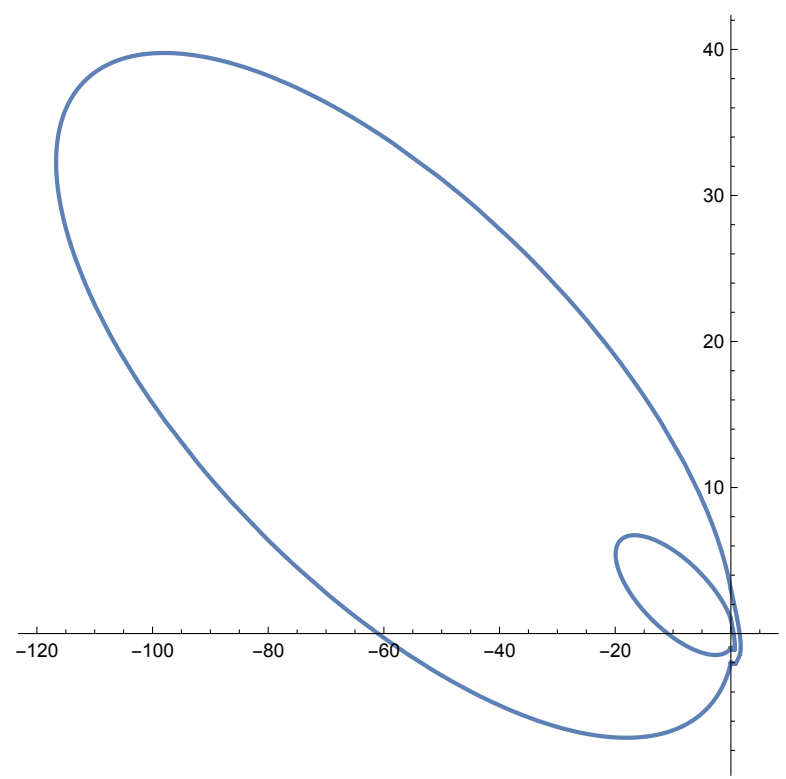

Figure 7: The two limit cycles that exist for system (25)-(26) of type (II)-(IV). They are travelled in counter-clockwise sense.

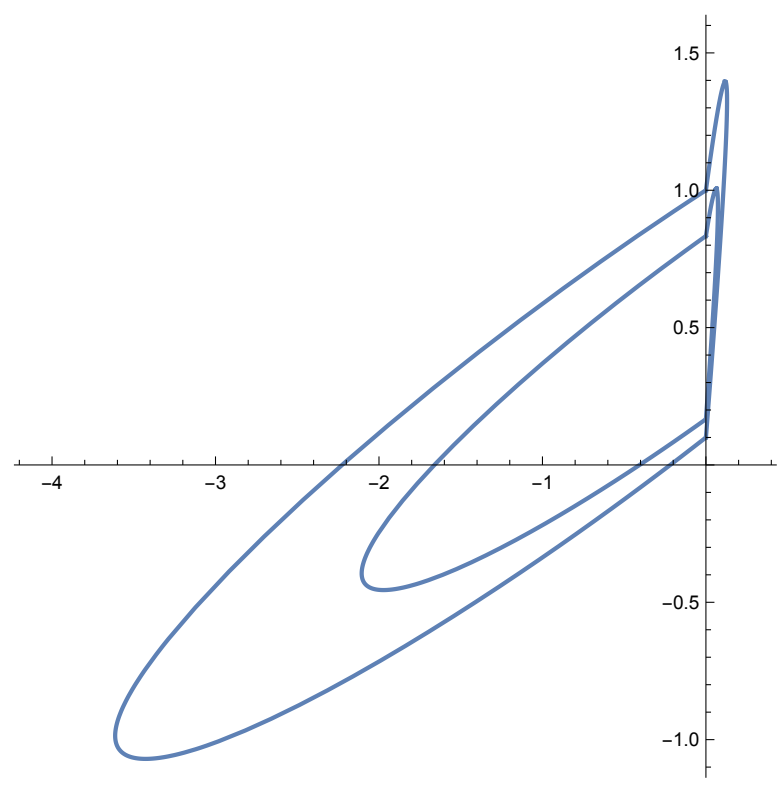

Figure 8: The two limit cycles existing for system (29)-(30) of type (II)-(V). They are travelled in counter-clockwise sense.

(b) at most three for systems of type (III)-(IV), and there are systems of this type with exactly two limit cycles, see Figure 10; 
(c) at most three for systems of type (III)-(V), and there are systems of this type with exactly two limit cycles, see Figure 9;

Note that for systems of type (III)-(III) after an affine change of variables the upper bound on the maximum number of limit cycles is reached.

The proof of Theorem 3 is given in section $\mathrm{V}$.

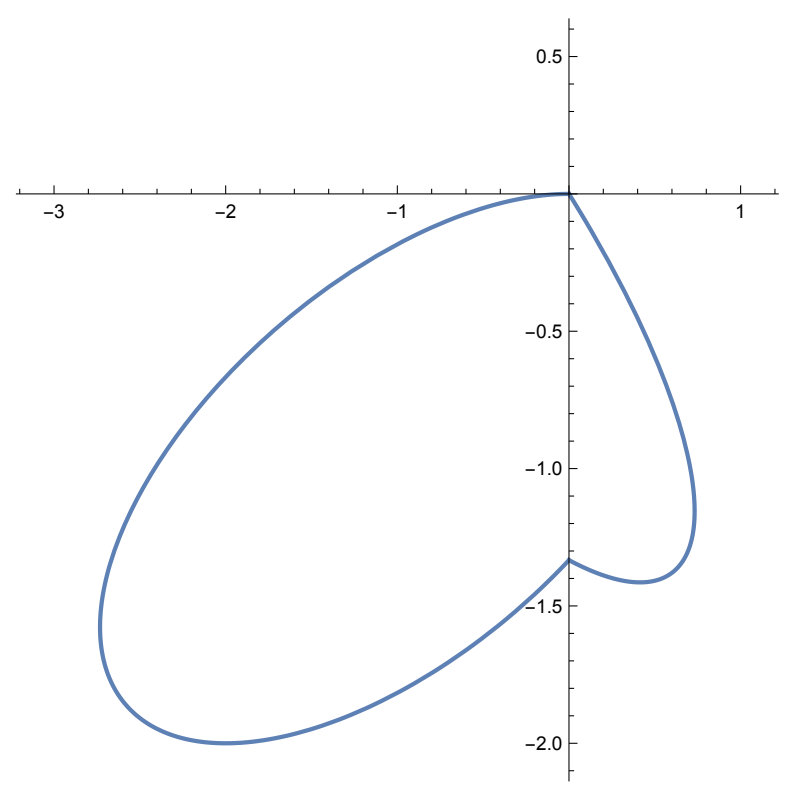

Figure 9: The unique limit cycle that exists for system (23)-(34) of type (III)-(III). It is travelled in counter-clockwise sense.

The following result gives the maximum number of limit cycles that can appear in discontinuous piecewise differential systems of the form (1) such that in a half-plane there is a general quadratic isochronous differential system of type (IV), and in the other one a general quadratic isochronous differential system of type (IV) or (V) after an arbitrary affine change of variables.

Theorem 4. Consider discontinuous piecewise differential systems separated by the straight line $x=0$ and formed by a quadratic isochronous center of type (IV) after an affine change of variables in $x<0$, and by a quadratic isochronous system of type either (IV), or (V) after an affine change of variables in $x>0$. The maximum number of limit cycles of these discontinuous piecewise differential systems are 


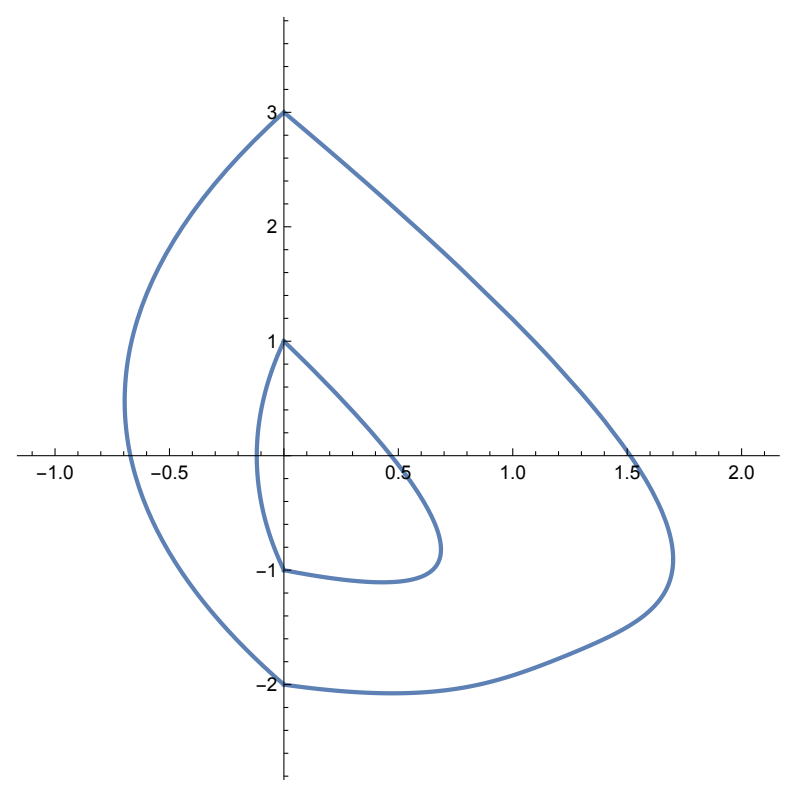

Figure 10: The pair of limit cycles that exists for system (36)-(26) of type (III)-(IV). They are travelled in counter-clockwise sense.

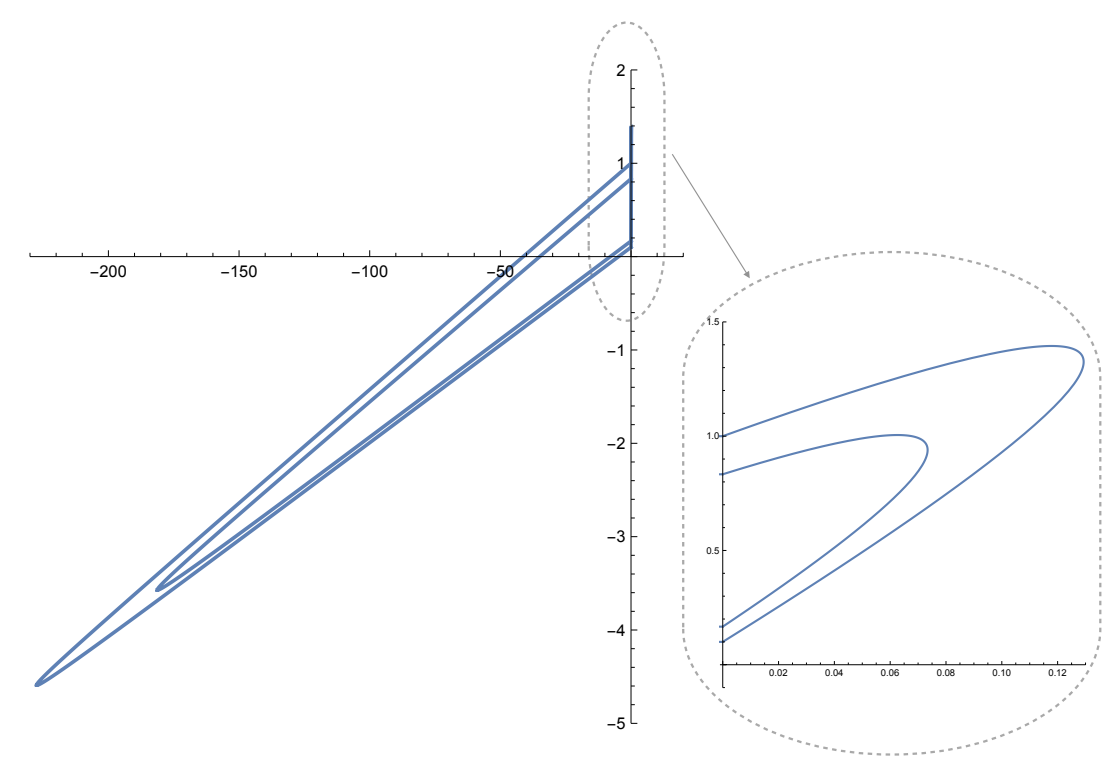

Figure 11: The two limit cycles existing for system (38)-(30) of type (III)-(V). They are travelled in counter-clockwise sense.

(a) at most three for systems of type (IV)-(IV), and there are systems of this type with 
exactly two limit cycles, see Figure 12;

(b) at most three for systems of type (IV)-(V), and there are systems of this type with exactly two limit cycle, see Figure 13.

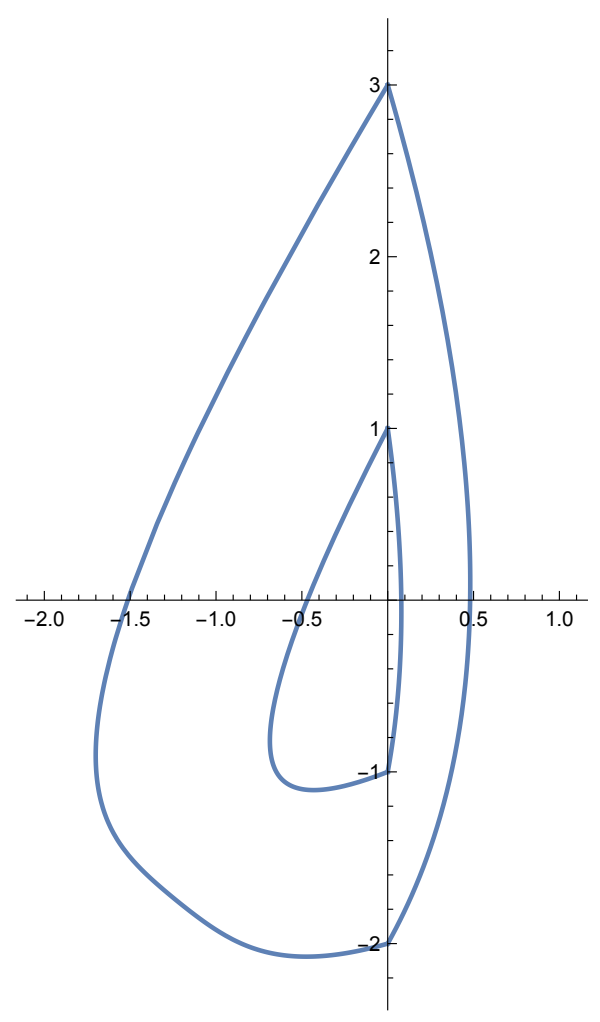

Figure 12: The pair of limit cycles that exists for system (41)-(43) of type (IV)-(IV). They are travelled in counter-clockwise sense.

The proof of Theorem 4 is given in section VI.

The last main result gives the maximum number of limit cycles that can appear in discontinuous piecewise differential systems of the form (1) such that in both half-planes there is a quadratic isochronous differential system of type (V) after an affine change of variables.

Theorem 5. The maximum number of limit cycles for discontinuous piecewise isochronous quadratic differential systems formed by two systems of type (V) separated by the straight line $x=0$ after an affine change of variables is at most twelve, and there are systems of this type with exactly two limit cycles, see Figure 14. 


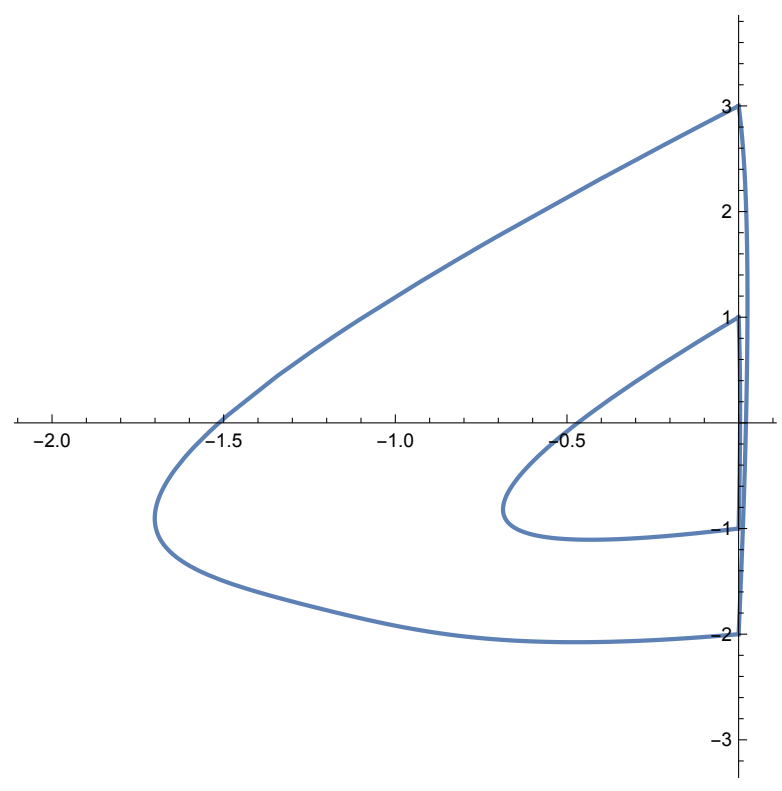

Figure 13: The two limit cycles existing for system (41)-(45) of type (IV)-(V). They are travelled in counter-clockwise sense.

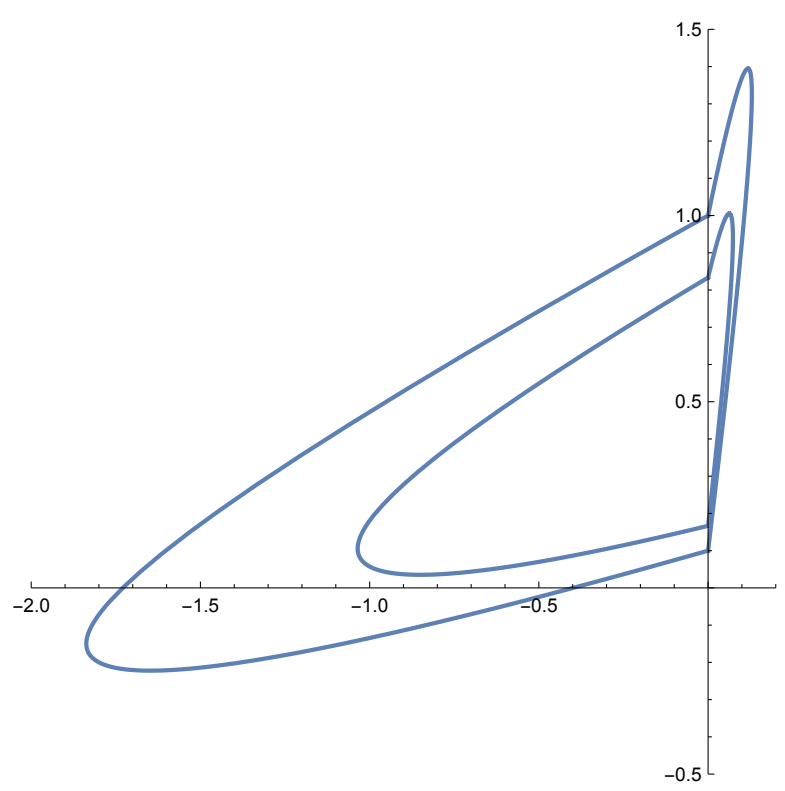

Figure 14: The two limit cycles existing for system (48)-(49) of type (V)-(V). They are travelled in counter-clockwise sense.

The proof of Theorem 5 is given in section VII. See the remark at the end of the proof of Theorem 5 related with this theorem. 


\section{THE QUADRATIC ISOCHRONOUS DIFFERENTIAL SYSTEMS (II), (III), (IV) AND (V) AFTER AN AFFINE CHANGE OF VARIABLES}

In this section we show the expressions for the quadratic isochronous systems (II), (III), (IV) and (V) and their first integrals, after doing the general affine change of variables of the form

$$
(x, y) \rightarrow(a x+b y+c, \alpha x+\beta y+\gamma)
$$

with $b \alpha-a \beta \neq 0$. Thus the differential system (II) after this affine change of variables becomes

$$
\begin{aligned}
\dot{x}= & \frac{1}{b \alpha-a \beta}\left(\beta \gamma^{2}+2 b \gamma c+b c+\beta \gamma-\beta c^{2}+(2 a b \gamma+2 \alpha \beta \gamma+a b+\alpha \beta\right. \\
& -2 a \beta c+2 \alpha b c) x+(2 \gamma+1)\left(b^{2}+\beta^{2}\right) y+\left(-a^{2} \beta+\alpha^{2} \beta+2 \alpha a b\right) x^{2} \\
& \left.+2 \alpha\left(b^{2}+\beta^{2}\right) x y+\beta\left(b^{2}+\beta^{2}\right) y^{2}\right), \\
\dot{y}= & \frac{1}{b \alpha-a \beta}\left(-\alpha \gamma^{2}-2 a \gamma c-a c-\alpha \gamma+\alpha c^{2}-(2 \gamma+1)\left(a^{2}+\alpha^{2}\right) x\right. \\
& +(-2 a b \gamma-2 \alpha \beta \gamma-a b-\alpha \beta-2 a \beta c+2 \alpha b c) y-\alpha\left(a^{2}+\alpha^{2}\right) x^{2} \\
& \left.-2 \beta\left(a^{2}+\alpha^{2}\right) x y-\left(\alpha \beta^{2}+2 a \beta b-\alpha b^{2}\right) y^{2}\right),
\end{aligned}
$$

whose first integral is

$$
H_{2}(x, y)=\frac{(c+a x+b y)^{2}+(x \alpha+y \beta+\gamma)^{2}}{1+2(x \alpha+y \beta+\gamma)}
$$

The differential system (III) becomes

$$
\begin{aligned}
\dot{x}= & \frac{1}{\alpha b-a \beta}\left(-b \gamma c-b c-\beta \gamma+\beta c^{2}+(-a b \gamma-a b-\alpha \beta+2 a \beta c\right. \\
& -\alpha b c) x-\left(b^{2} \gamma+b^{2}+\beta^{2}+\beta b c\right) y+a(a \beta-\alpha b) x^{2} \\
& -b(\alpha b-a \beta) x y), \\
\dot{y}= & \frac{1}{\alpha b-a \beta}\left(-a \gamma c-a c-\alpha \gamma+\alpha c^{2}-\left(a^{2} \gamma+a^{2}+\alpha^{2}-\alpha a c\right) x\right. \\
& +(-a b \gamma-a b-\alpha \beta-a \beta c+2 \alpha b c) y-a(a \beta-\alpha b) x y \\
& \left.+b(\alpha b-a \beta) y^{2}\right)
\end{aligned}
$$


whose first integral is

$$
H_{3}(x, y)=\frac{(a x+b y+c)^{2}+(\gamma+\alpha x+\beta y)^{2}}{(\gamma+\alpha x+\beta y+1)^{2}}
$$

The differential system (IV) becomes,

$$
\begin{aligned}
\dot{x}= & \frac{1}{3(\alpha b-a \beta)}\left(-16 b \gamma c+3 b c+3 \beta \gamma+4 \beta c^{2}+(-16 a b \gamma+3 a b\right. \\
& +3 \alpha \beta+8 a \beta c-16 \alpha b c) x+\left(-16 b^{2} \gamma+3 b^{2}+3 \beta^{2}-8 \beta b c\right) y \\
& \left.+4 a(a \beta-4 \alpha b) x^{2}-8 b(a \beta+2 \alpha b) x y-12 b^{2} \beta y^{2}\right), \\
\dot{y}= & \frac{1}{3(\alpha b-a \beta)}\left(16 a \gamma c-3 a c-3 \alpha \gamma-4 \alpha c^{2}+\left(16 a^{2} \gamma-3 a^{2}\right.\right. \\
& \left.-3 \alpha^{2}+8 \alpha a c\right) x+(16 a b \gamma-3 a b-3 \alpha \beta+16 a \beta c-8 \alpha b c) y \\
& \left.+12 a^{2} \alpha x^{2}+8 a(2 a \beta+\alpha b) x y-4 b(\alpha b-4 a \beta) y^{2}\right)
\end{aligned}
$$

whose first integral is

$$
\begin{aligned}
H_{4}(x, y)= & \frac{1}{16(\gamma+\alpha x+\beta y)-3}\left(-24(a x+b y+c)^{2}(\gamma+\alpha x+\beta y)\right. \\
& \left.+9\left((a x+b y+c)^{2}+(\gamma+\alpha x+\beta y)^{2}\right)+16(a x+b y+c)^{4}\right)
\end{aligned}
$$

Finally the differential system (V) after the change of variables (2) becomes

$$
\begin{aligned}
\dot{x}= & \frac{1}{3(\alpha b-a \beta)}\left(4 \beta \gamma^{2}+8 b \gamma c+3 b c+3 \beta \gamma-16 \beta c^{2}+(8 a b \gamma+8 \alpha \beta \gamma\right. \\
& +3 a b+3 \alpha \beta-32 a \beta c+8 \alpha b c) x+\left(8 b^{2} \gamma+8 \beta^{2} \gamma+3 b^{2}+3 \beta^{2}\right. \\
& -24 \beta b c) y+4\left(\alpha^{2} \beta-4 a^{2} \beta+2 \alpha a b\right) x^{2}+8\left(\alpha \beta^{2}-3 a \beta b+\alpha b^{2}\right) x y \\
& \left.-4 \beta y^{2}\left(2 b^{2}-\beta^{2}\right) y^{2}\right) \\
\dot{y}= & \frac{1}{3(\alpha b-a \beta)}\left(16 \alpha c^{2}-4 \alpha \gamma^{2}-8 a \gamma c-3 a c-3 \alpha \gamma-\left(8 a^{2} \gamma+8 \alpha^{2} \gamma\right.\right. \\
& \left.+3 a^{2}+3 \alpha^{2}-24 \alpha a c\right) x-(8 a b \gamma+8 \alpha \beta \gamma+3 a b+3 \alpha \beta+8 a \beta c \\
& -32 \alpha b c) y+4 \alpha\left(2 a^{2}-\alpha^{2}\right) x^{2}+8\left(a^{2}(-\beta)-\alpha^{2} \beta+3 \alpha a b\right) x y \\
& \left.-4\left(\alpha \beta^{2}+2 a \beta b-4 \alpha b^{2}\right) y^{2}\right)
\end{aligned}
$$


whose first integral is

$$
\begin{aligned}
H_{5}(x, y)= & \frac{1}{(8(\gamma+\alpha x+\beta y)+3)^{4}}\left(9\left((a x+b y+c)^{2}+(\gamma+\alpha x+\beta y)^{2}\right)\right. \\
& \left.+16(\gamma+\alpha x+\beta y)^{4}+24(\gamma+\alpha x+\beta y)^{3}\right) .
\end{aligned}
$$

\section{PROOF OF THEOREM 1}

\section{A. Proof of Theorem 1 for system (I)-(II)}

We consider the planar linear differential system (I) with first integral $H_{1}(x, y)$ in the half-plane $x<0$ and the quadratic polynomial differential system (3) with first integral $H_{2}(x, y)$ in the half-plane $x>0$. If there exists a limit cycle of the discontinuous piecewise differential systems (I)-(3) it must intersect the discontinuity line $x=0$ in two different points $(0, y)$ and $(0, Y)$. Clearly these two points must satisfy the system

$$
\begin{aligned}
H_{1}(0, y)-H_{1}(0, Y) & =(Y-y)\left(-4 A^{2} y-4 A^{2} Y+8 B-y \omega^{2}-\omega^{2} Y\right) \\
& =(Y-y) P_{1}(y, Y)=0 \\
H_{2}(0, y)-H_{2}(0, Y) & =\frac{(Y-y) Q_{2}(y, Y)}{[1+2(\beta y+\gamma)][1+2(\beta Y+\gamma)]}=0
\end{aligned}
$$

where $P_{1}$ and $Q_{2}$ are polynomials of degrees one and two, respectively. Since the points $(0, y)$ and $(0, Y)$ are different, from $P_{1}(y, Y)=0$, we get $Y$ as a function of $y$, that is, $Y=f(y)$. Substituting this expression in equation $Q_{2}(y, Y)=0$ we obtain a quadratic equation in the variable $y$. Then the maximum number of solutions of (7) is two, namely $\left(y_{1}, Y_{1}\right)$ and $\left(y_{2}, Y_{2}\right)$, but in fact, these two solutions represent the same limit cycle because $Y_{1}=y_{2}$ and $Y_{2}=y_{1}$. So for the discontinuous piecewise differential system (I)-(3), there exists at most one limit cycle.

Now we give an example of a discontinuous piecewise differential system (I)-(3) having one limit cycle. On $x>0$ we consider the linear differential system

$$
\dot{x}=1-x-\frac{5}{4} y, \quad \dot{y}=x+y,
$$


whose first integral is

$$
H_{1}(x, y)=-8 y+y^{2}+4(x+y)^{2}
$$

and on $x>0$ we consider the quadratic isochronous differential system of type (3)

$$
\dot{x}=-4-5 x-6 y-x^{2}-4 x y-2 y^{2}, \quad \dot{y}=1+3 x+y+x^{2}+2 x y,
$$

whose first integral is

$$
H_{2}(x, y)=\frac{(x+y+1)^{2}+(y+1)^{2}}{2(x+y+1)+1} .
$$

We can take, without loss of generality, the solution of (7) satisfying $y<Y$, and so the pair $(y, Y)=\left(\frac{1}{5}(4-3 \sqrt{14}), \frac{1}{5}(4+3 \sqrt{14})\right)$ provides the limit cycle that exists for the discontinuous differential piecewise system (8)-(9) shown in Figure 1.

\section{B. Proof of Theorem 1 for system (I)-(III)}

We consider the linear differential system (I) with first integral $H_{1}(x, y)$ on the halfplane $x<0$, and on the half-plane $x>0$, we take the quadratic isochronous differential system (4) with its first integral $H_{3}(x, y)$. Then if there exists some limit cycle for the discontinuous differential system (I)-(4), it must intersect the discontinuity line $x=0$ at two different points $(0, y)$ and $(0, Y)$, satisfying the equations

$$
\begin{aligned}
& H_{1}(0, y)-H_{1}(0, Y)=(Y-y) P_{1}(y, Y)=0 \\
& H_{3}(0, y)-H_{3}(0, Y)=\frac{(Y-y) Q_{3}(y, Y)}{(1+\beta y+\gamma)^{2}(1+\beta Y+\gamma)^{2}}=0
\end{aligned}
$$

In (10) $P_{1}$ and $Q_{3}$ are polynomials of degrees one and two, respectively. By following the same procedure as for the proof of system (I)-(II), we solve the equation $P_{1}(y, Y)=0$ obtaining the variable $Y$ as a function of $y$, that is $Y=f(y)$. By replacing $Y$ in the equation $Q_{3}(y, Y)=0$, we obtain again a quadratic polynomial equation in the variable $y$, so that the equation has at most two different solutions. As in the proof for system (I)-(II), these two solutions represent, if they exist, the same limit cycle. Therefore system (10) has only one solution with $y<Y$, and then the discontinuous piecewise 
differential system (I)-(4) has at most one limit cycle.

Next we give a specific discontinuous piecewise differential system (I)-(4) having one limit cycle. On the half-plane $x<0$ we consider the linear differential system (8), and on the half-plane $x>0$ we consider the quadratic isochronous differential system of type (4)

$$
\dot{x}=-2-2 y+x^{2}+x y, \quad \dot{y}=2+2 x+3 y+x y+y^{2},
$$

with first integral

$$
H_{3}(x, y)=\frac{(x+y+1)^{2}+(y+1)^{2}}{(y+2)^{2}} .
$$

In this case, the unique solution for system (10) with $y<Y$ is

$$
(y, Y)=\left(\frac{1}{5}(4-3 \sqrt{14}), \frac{1}{5}(4+3 \sqrt{14})\right)
$$

and the corresponding limit cycle of the discontinuous piecewise differential system (8)(11) associated to this solution is shown in Figure 2.

\section{Proof of Theorem 1 for system (I)-(IV)}

We consider again on the half-plane $x<0$ the linear differential system (I) with its first integral $H_{1}(x, y)$, and on $x>0$ we take the quadratic isochronous differential system (5) with its first integral $H_{4}(x, y)$. Then if the discontinuous differential system (I)-(5) has a limit cycle, it must intersect the discontinuity line $x=0$ at two different points $(0, y)$ and $(0, Y)$. These points must satisfy the equations

$$
\begin{aligned}
& H_{1}(0, y)-H_{1}(0, Y)=(Y-y) P_{1}(y, Y)=0 \\
& H_{4}(0, y)-H_{4}(0, Y)=\frac{(Y-y) Q_{4}(y, Y)}{(-3+16 y \beta+16 \gamma)(-3+16 Y \beta+16 \gamma)}=0
\end{aligned}
$$

where $P_{1}$ and $Q_{4}$ are polynomials of degrees one and four, respectively. We solve the equation $P_{1}(y, Y)=0$ obtaining the variable $Y$ as a function of $y$, that is $Y=f(y)$. If we substitute $Y=f(y)$ in the equation $Q_{4}(y, Y)=0$, we obtain a polynomial equation of degree four in the variable $y$, and so system (12) has at most four real solutions. Taking 
into account the symmetry between these solutions, as in the previous statements there can be only two different solutions $(y, Y)$ of (12) satisfying $y<Y$.

Now, we show a concrete discontinuous piecewise differential system (I)-(5) having a unique limit cycle. On the half-plane $x<0$ we consider the linear differential system

$$
\dot{x}=1-x-2 y, \quad \dot{y}=x+y,
$$

with first integral

$$
H_{1}(x, y)=-2 y+y^{2}+(x+y)^{2},
$$

and on the half-plane $x>0$ we consider the quadratic isochronous differential system of type (4) given by

$$
\begin{aligned}
& \dot{x}=-8.98889-17.5383 x-20.7447 y-2.21606 x^{2}-9.76545 x y-7.54939 y^{2}, \\
& \dot{y}=14.6779+21.205 x+25.5383 y+3.54939 x^{2}+12.4321 x y+8.88273 y^{2},
\end{aligned}
$$

with first integral

$$
\begin{aligned}
H_{4}(x, y)= & \frac{1}{16 x+0.0318943(1067 y+2091)}\left(16(1+x+y)^{4}\right. \\
& -24(1+x+y)^{2}(4.35569+x+2.12695 y)+9\left((1+x+y)^{2}\right. \\
& \left.\left.+(4.35569+x+2.12695 y)^{2}\right)\right) .
\end{aligned}
$$

In this case the solution for system (12) with $y<Y$ is

$$
\left(y_{1}, Y_{1}\right)=(-1.898651543493539,2.898651543493539)
$$

and the corresponding limit cycle of the discontinuous piecewise differential system (13)(14) associated to these solutions are shown in Figure 3.

Remark. For all these discontinuous piecewise differential systems it is possible that the upper bound found for the maximum number of limit cycles cannot be reached. This is due to the fact that the solutions $(y, Y)$ do not need to correspond necessarily to periodic solutions of the discontinuous piecewise differential systems. 


\section{Proof of Theorem 1 for system (I)-(V)}

We take again the linear differential system (I) with its first integral $H_{1}(x, y)$ on the half-plane $x<0$, and on $x>0$ we consider the quadratic isochronous differential system (6) with its first integral $H_{5}(x, y)$. Thus if the discontinuous differential system (I)-(6) has a limit cycle, it must intersect the discontinuity line $x=0$ at two different points $(0, y)$ and $(0, Y)$. These points must satisfy the equations

$$
\begin{aligned}
& H_{1}(0, y)-H_{1}(0, Y)=(Y-y) P_{1}(y, Y)=0 \\
& H_{5}(0, y)-H_{5}(0, Y)=\frac{(Y-y) Q_{5}(y, Y)}{(3+8 y \beta+8 \gamma)^{4}(3+8 Y \beta+8 \gamma)^{4}}=0,
\end{aligned}
$$

where $P_{1}$ and $Q_{5}$ are polynomials of degrees one and five, respectively. We solve again the equation $P_{1}(y, Y)=0$ obtaining the variable $Y$ as a function of $y$, that is $Y=f(y)$. If we substitute $Y=f(y)$ in the equation $Q_{5}(y, Y)=0$, we obtain a polynomial equation of degree 4 in the variable $y$, and so system (12) has at most four real solutions. Taking into account the symmetry between these solutions, as in the previous statements, there can be only two different solutions $(y, Y)$ of $(15)$ satisfying $y<Y$.

Finally we show a discontinuous piecewise differential system (I)-(6) having two limit cycles. On the half-plane $x<0$ we consider the linear differential system

$$
\dot{x}=1+x-2 y, \quad \dot{y}=x-y,
$$

with first integral

$$
H_{1}(x, y)=-2 y+x^{2}-2 x y+2 y^{2},
$$

and on the half-plane $x>0$ we consider the quadratic isochronous differential system of type $(6)$

$$
\begin{aligned}
& \dot{x}=0.00134583+7.88817 x+0.03269 y-5.33333 x^{2}+5.81077 x y-0.0735418 y^{2}, \\
& \dot{y}=3.82531-3.82531 x+5.44516 y-2.66667 x y+1.93692 y^{2},
\end{aligned}
$$


with first integral

$$
\begin{aligned}
H_{5}(x, y)= & \frac{1}{(8(-1 y-1.80949)+3)^{4}}\left(9\left((-1 x+0.726346 y+1)^{2}+(-1 y-1.80949)^{2}\right)\right. \\
& \left.+16(-1 y-1.80949)^{4}+24 .(-1 y-1.80949)^{3}\right) .
\end{aligned}
$$

For this case, the two solutions for system (15) with $y<Y$ are

$$
\left(y_{1}, Y_{1}\right)=(0,1), \quad\left(y_{2}, Y_{2}\right)=\left(\frac{1}{5}, \frac{4}{5}\right)
$$

and the corresponding limit cycles of the discontinuous piecewise differential system (8)-(17) associated to this solution is shown in Figure 4.

\section{PROOF OF THEOREM 2}

\section{A. Proof of statement (a) of Theorem 2.}

We consider the quadratic polynomial differential system (3) with first integral $H_{2}(x, y)$ in the half-plane $x<0$. By changing the parameters $(a, \alpha, b, \beta, c, \gamma)$ to $\left(a_{1}, \alpha_{1}, b_{1}, \beta_{1}, c_{1}, \gamma_{1}\right)$ in system (3) and in its first integral, we obtain a second isochronous quadratic differential system of type (3) with the first integral $\widetilde{H}_{2}(x, y)$, namely

$$
\begin{aligned}
& \dot{x}=\frac{1}{b_{1} \alpha_{1}-a_{1} \beta_{1}}\left(\beta_{1} \gamma_{1}^{2}+2 b_{1} \gamma_{1} c_{1}+b_{1} c_{1}+\beta_{1} \gamma_{1}-\beta_{1} c_{1}^{2}+\left(2 a_{1} b_{1} \gamma_{1}+2 \alpha_{1} \beta_{1} \gamma_{1}\right.\right. \\
& \left.+a_{1} b_{1}+\alpha_{1} \beta_{1}-2 a_{1} \beta_{1} c_{1}+2 \alpha_{1} b_{1} c_{1}\right) x+\left(2 \gamma_{1}+1\right)\left(b_{1}^{2}+\beta_{1}^{2}\right) y+\left(-a_{1}^{2} \beta_{1}+\alpha_{1}^{2} \beta_{1}\right. \\
& \left.\left.+2 \alpha_{1} a_{1} b_{1}\right) x^{2}+2 \alpha_{1}\left(b_{1}^{2}+\beta_{1}^{2}\right) x y+\beta_{1}\left(b_{1}^{2}+\beta_{1}^{2}\right) y^{2}\right), \\
& \dot{y}=\frac{1}{b_{1} \alpha_{1}-a_{1} \beta_{1}}\left(-\alpha_{1} \gamma_{1}^{2}-2 a_{1} \gamma_{1} c_{1}-a_{1} c_{1}-\alpha_{1} \gamma_{1}+\alpha_{1} c_{1}^{2}-\left(2 \gamma_{1}+1\right)\left(a_{1}^{2}+\alpha_{1}^{2}\right) x\right. \\
& +\left(-2 a_{1} b_{1} \gamma_{1}-2 \alpha_{1} \beta_{1} \gamma_{1}-a_{1} b_{1}-\alpha_{1} \beta_{1}-2 a_{1} \beta_{1} c_{1}+2 \alpha_{1} b_{1} c_{1}\right) y-\alpha_{1}\left(a_{1}^{2}+\alpha_{1}^{2}\right) x^{2} \\
& \left.-2 \beta_{1}\left(a_{1}^{2}+\alpha_{1}^{2}\right) x y-\left(\alpha_{1} \beta_{1}^{2}+2 a_{1} \beta_{1} b_{1}-\alpha_{1} b_{1}^{2}\right) y^{2}\right),
\end{aligned}
$$

whose first integral is

$$
\widetilde{H}_{2}(x, y)=\frac{\left(c_{1}+a_{1} x+b_{1} y\right)^{2}+\left(x \alpha_{1}+y \beta_{1}+\gamma_{1}\right)^{2}}{1+2\left(x \alpha_{1}+y \beta_{1}+\gamma_{1}\right)} .
$$


If a limit cycle of the discontinuous piecewise differential system (3)-(18) has two different intersection points $(0, y)$ and $(0, Y)$ with the line $x=0$, then they must satisfy the system

$$
\begin{aligned}
& H_{2}(0, y)-H_{2}(0, Y)=\frac{(Y-y) P_{2}(y, Y)}{(2 \gamma+2 \beta y+1)(2 \gamma+2 \beta Y+1)}=0, \\
& \widetilde{H}_{2}(0, y)-\widetilde{H}_{2}(0, Y)=\frac{(Y-y) Q_{2}(y, Y)}{\left(2 \gamma_{1}+2 \beta_{1} y+1\right)\left(2 \gamma_{1}+2 \beta_{1} Y+1\right)}=0,
\end{aligned}
$$

where both polynomials $P_{2}$ and $Q_{2}$ are of degree two. From the equation $Q_{2}(y, Y)=0$, we get $Y$ as a function of $y$, that is, $Y=f(y)$, and substituting this expression in the equation $P_{2}(y, Y)=0$, we obtain a quadratic polynomial equation in the variable $y$. Then the maximum number of solutions of (19) is two, namely $\left(y_{1}, Y_{1}\right)$ and $\left(y_{2}, Y_{2}\right)$, but in fact, these two solutions represent the same limit cycle because they are symmetric in the sense of the proof of Theorem 1. Thus system (3)-(3) has at most one limit cycle.

Now we give an example of a discontinuous piecewise differential system of type (3)(3) having one limit cycle. On $x<0$ we consider the quadratic isochronous differential system

$$
\dot{x}=-4-x-6 y+x^{2}-2 y^{2}, \quad \dot{y}=3+3 x+5 y+2 x y+2 y^{2},
$$

with a first integral

$$
H_{2}(x, y)=\frac{(1+y)^{2}+(1+x+y)^{2}}{1+2(1+y)}
$$

and on $x>0$ we consider the quadratic isochronous differential system of type (3)

$$
\dot{x}=-2+x-6 y+x^{2}-4 x y+2 y^{2}, \quad \dot{y}=1+3 x-5 y+x^{2}-2 x y,
$$

whose first integral is

$$
\widetilde{H}_{2}(x, y)=\frac{(1+x-y)^{2}+(1+y)^{2}}{1+2(1+x-y)}
$$

The solution of (19) satisfying $y<Y$ is $(y, Y)=\left(\frac{1}{2}(-1-\sqrt{3}), \frac{1}{2}(-1+\sqrt{3})\right)$, which provides the limit cycle for the discontinuous differential piecewise system (20)-(21) shown in Figure 5. 


\section{B. Proof of statement (b) of Theorem 2.}

We consider again the quadratic polynomial differential system (18) with first integral $\widetilde{H}_{2}(x, y)$ in the half-plane $x<0$ and for $x>0$ we take the isochronous differential system (4) whose first integral is $H_{3}(x, y)$.

If there exists a limit cycle of the discontinuous piecewise differential system (18)-(4), then it has two different intersection points $(0, y)$ and $(0, Y)$ with the line $x=0$, which satisfy the system

$$
\begin{aligned}
\widetilde{H}_{2}(0, y)-\widetilde{H}_{2}(0, Y) & =\frac{(Y-y) P_{2}(y, Y)}{\left(2 \gamma_{1}+2 \beta_{1} y+1\right)\left(2 \gamma_{1}+2 \beta_{1} Y+1\right)}=0, \\
H_{3}(0, y)-H_{3}(0, Y) & =\frac{(Y-y) Q_{2}(y, Y)}{\left(\gamma_{2}+\beta_{2} y+1\right)^{2}\left(\gamma_{2}+\beta_{2} Y+1\right)^{2}}=0,
\end{aligned}
$$

where both polynomials $P_{2}$ and $Q_{2}$ have degree two. From the equation $P_{2}(y, Y)=0$, we get $Y$ as a function of $y$, that is, $Y=f(y)$, and substituting this expression in the equation $Q_{2}(y, Y)=0$, we obtain a polynomial equation of degree two in the variable $y$. Then the maximum number of solutions of (22) is two. But due to the symmetry of the solutions, the system (3)-(4) has at most one limit cycle.

Now we write an example of a discontinuous piecewise differential system having a unique limit cycle. On $x<0$ we consider the quadratic isochronous differential system (20), and on $x>0$ we consider the quadratic isochronous differential system of type (4)

$$
\dot{x}=-2-4 y-x y, \quad \dot{y}=x-3 y-y^{2},
$$

whose first integral is

$$
H_{3}(x, y)=\frac{(x-y+1)^{2}+(y+1)^{2}}{(x-y+2)^{2}}
$$

Then, the obtained solution of system (22) satisfying $y<Y$ is $(y, Y)=\left(-\frac{4}{3}, 0\right)$. This pair, provides the limit cycle that exists for the discontinuous differential piecewise system (20)-(23) shown in Figure 6. 


\section{Proof of statement (c) of Theorem 2.}

We take the quadratic polynomial differential system (18) with the first integral $\widetilde{H}_{2}(x, y)$ in the half-plane $x<0$ and for $x>0$ we take the isochronous differential system (5) with the first integral $H_{4}(x, y)$.

If there exists a limit cycle of the discontinuous piecewise differential system (18)-(5), then it has two different intersection points $(0, y)$ and $(0, Y)$ with the separation line $x=0$, satisfying the system

$$
\begin{aligned}
\widetilde{H}_{2}(0, y)-\widetilde{H}_{2}(0, Y) & =\frac{(Y-y) P_{2}(y, Y)}{\left(2 \gamma_{1}+2 \beta_{1} y+1\right)\left(2 \gamma_{1}+2 \beta_{1} Y+1\right)}=0 \\
H_{4}(0, y)-H_{4}(0, Y) & =\frac{(Y-y) Q_{4}(y, Y)}{(-3+16 y \beta+16 \gamma)(-3+16 Y \beta+16 \gamma)}=0
\end{aligned}
$$

where both polynomials $P_{2}$ and $Q_{4}$ are of degree two and four respectively. From the equation $P_{2}(y, Y)=0$, we obtain $Y$ as a function of $y$, that is, $Y=f(y)$, and if we put this expression in the second equation $Q_{4}(y, Y)=0$, we obtain a polynomial equation of degree six in the variable $y$. Then, the maximum number of solutions of (22) is six, but due to the symmetry, there are at most three solutions of system (24) satisfying $y<Y$. Thus system (3)-(5) has at most three limit cycles.

Next we give an example of discontinuous piecewise differential system of type (3)-(5) having two limit cycles. On $x<0$ we consider the quadratic isochronous differential system

$$
\begin{aligned}
& \dot{x}=-0.427384-0.457523 x-4.27384 y+1.9317 x^{2}+6.83814 x y-0.427384 y^{2}, \\
& \dot{y}=0.116463+0.68296 x+1.87174 y-0.546368 x^{2}+0.136592 x y+3.652 y^{2}
\end{aligned}
$$

with the first integral

$$
\widetilde{H}_{2}(x, y)=\frac{9+128 x^{2}+9 y(18+89 y)-16 x(1-2 \sqrt{2}+y-20 \sqrt{2} y)}{16(5-8 x+y)}
$$


and on $x>0$ we consider the quadratic isochronous differential system of type (5)

$$
\begin{aligned}
& \dot{x}=-0.593985-6.89377 x-6.77032 y+3.03414 x^{2}+0.667964 x y-1.89916 y^{2}, \\
& \dot{y}=4.77286+7.53417 x+9.75481 y-1.87138 x^{2}+1.93171 x y+3.30143 y^{2},
\end{aligned}
$$

whose first integral is

$$
\begin{aligned}
H_{4}(x, y)= & \frac{1}{54.173-16 x+19.6577 y}\left(16(-0.35763-x-0.908852 y)^{4}\right. \\
& -24(-0.35763-x-0.908852 y)^{2}(3.57331-x+1.22861 y) \\
& \left.+9\left((-0.35763-x-0.908852 y)^{2}+(3.57331-x+1.22861 y)^{2}\right)\right) .
\end{aligned}
$$

The solutions of (24) satisfying $y<Y$ are

$$
\left(y_{1}, Y_{1}\right)=(-1,1), \quad\left(y_{2}, Y_{2}\right)=(-2,3)
$$

which provide the two limit cycles for the discontinuous differential piecewise system (25)-(26) shown in Figure 7.

\section{Proof of statement (d) of Theorem 2 .}

We consider the quadratic polynomial differential system (18) with the first integral $\widetilde{H}_{2}(x, y)$ in the half-plane $x<0$, and for $x>0$ we take the isochronous differential system (6) whose first integral is $H_{5}(x, y)$.

If a limit cycle exists for the discontinuous piecewise differential system (18)-(6), then it has two different intersection points $(0, y)$ and $(0, Y)$ with the line $x=0$, satisfying the closing equations

$$
\begin{aligned}
\widetilde{H}_{2}(0, y)-\widetilde{H}_{2}(0, Y) & =\frac{(Y-y) P_{2}(y, Y)}{\left(2 \gamma_{1}+2 \beta_{1} y+1\right)\left(2 \gamma_{1}+2 \beta_{1} Y+1\right)}=0, \\
H_{5}(0, y)-H_{5}(0, Y) & =\frac{(Y-y) Q 5(y, Y)}{(8 \gamma+8 \beta y+3)(8 \gamma+8 \beta Y+3)}=0,
\end{aligned}
$$

where both polynomials $P_{2}$ and $Q_{5}$ are of degree two and five respectively. From the equation $P_{2}(y, Y)=0$, we get $Y$ as a function of $y$, that is, $Y=f(y)$, and substituting 
this expression in the equation $Q_{5}(y, Y)=0$, we obtain an equation of order six in the variable $y$. Then, the maximum number of solutions of (28) is six, but because of the symmetry property as in the previous statements, system (3)-(6) has three limit cycle at most. Next, we give an example of discontinuous piecewise differential system of type (3)-(6) having two limit cycles. On $x<0$ we consider the quadratic isochronous differential system

$$
\begin{aligned}
& \dot{x}=-4.24371+0.170949 x+6.25388 y+3.65748 x^{2}-12.5078 x y+8.04071 y^{2}, \\
& \dot{y}=-2.20072-1.28915 x+5.48591 y+1.28915 x^{2}-3.31496 x y+0.579633 y^{2},
\end{aligned}
$$

with the first integral

$$
\widetilde{H}_{2}(x, y)=\frac{14\left(1.5+x^{2}+x(0.414214-4.12284 y)+y(-2.72659+4.85117 y)\right)}{-7+14 x-18 y},
$$

and on $x>0$ we consider the quadratic isochronous differential system of type (6)

$$
\begin{aligned}
& \dot{x}=0.550125+9.00984 x-1.24812 y+6.05959 x^{2}-0.600689 x y-0.03414 y^{2}, \\
& \dot{y}=3.90588+49.7765 x-6.63534 y+17.3476 x^{2}+1.21415 x y-0.491153 y^{2},
\end{aligned}
$$

whose first integral is

$$
\begin{aligned}
H_{5}(x, y)= & \frac{0.000244141}{\left.(-0.450206+x-0.195584 y)^{4}\right)}\left(9 \left((-0.825206+x-0.195584 y)^{2}\right.\right. \\
& \left.+(0.178088+x-0.118725 y)^{2}\right)+24(-0.825206+x-0.195584 y)^{3} \\
& \left.+16(-0.825206+x-0.195584 y)^{4}\right) .
\end{aligned}
$$

The solutions of (28) satisfying $y<Y$ which provide the two limit cycles for the discontinuous differential piecewise system (29)-(30) are

$$
\left(y_{1}, Y_{1}\right)=(0.1 ., 1 .), \quad\left(y_{2}, Y_{2}\right)=(0.166666,0.833335)
$$

shown in Figure 8. 


\section{PROOF OF THEOREM 3}

\section{A. Proof of statement (a) of Theorem 3.}

We consider the quadratic polynomial differential system (4) with first integral $H_{3}(x, y)$ in the half-plane $x<0$. By changing the parameters $(a, \alpha, b, \beta, c, \gamma)$ to $\left(a_{1}, \alpha_{1}, b_{1}, \beta_{1}, c_{1}, \gamma_{1}\right)$ in system (4) and in its first integral, we obtain a second isochronous quadratic differential system of type (4) with the first integral $\widetilde{H}_{3}(x, y)$, namely

$$
\begin{aligned}
& \dot{x}=\frac{1}{b_{1} \alpha_{1}-a_{1} \beta_{1}}\left(b_{1}^{2} y\left(1+x \alpha_{1}+\gamma_{1}\right)+b_{1}\left(c_{1}+a_{1} x\right)\left(1+x \alpha_{1}-y \beta_{1}+\gamma_{1}\right)\right. \\
& \left.+\beta_{1}\left(-c_{1}^{2}-2 a_{1} c_{1} x-a_{1}^{2} x^{2}+x \alpha_{1}+y \beta_{1}+\gamma_{1}\right)\right) \\
& \dot{y}=\frac{1}{b_{1} \alpha_{1}-a_{1} \beta_{1}}\left(\alpha_{1}\left(c_{1}^{2}+2 b_{1} c_{1} y+b_{1}^{2} y^{2}-x \alpha_{1}-y \beta_{1}-\gamma_{1}\right)-a_{1}^{2} x\left(1+y \beta_{1}+\gamma_{1}\right)\right. \\
& \left.-a_{1}\left(c_{1}+b_{1} y\right)\left(1-x \alpha_{1}+y \beta_{1}+\gamma_{1}\right)\right),
\end{aligned}
$$

whose first integral is

$$
\widetilde{H}_{3}(x, y)=\frac{\left(c_{1}+a_{1} x+b_{1} y\right)^{2}+\left(x \alpha_{1}+y \beta_{1}+\gamma_{1}\right)^{2}}{\left(1+x \alpha_{1}+y \beta_{1}+\gamma_{1}\right)^{2}} .
$$

If a limit cycle of the discontinuous piecewise differential system (4)-(32) has two different intersection points $(0, y)$ and $(0, Y)$ with the line $x=0$, then they must satisfy the system

$$
\begin{aligned}
& H_{3}(0, y)-H_{3}(0, Y)=\frac{(Y-y) P_{2}(y, Y)}{(1+y \beta+\gamma)^{2}(1+Y \beta+\gamma)^{2}}=0 \\
& \widetilde{H}_{3}(0, y)-\widetilde{H}_{3}(0, Y)=\frac{(Y-y) Q_{2}(y, Y)}{\left(1+y \beta_{1}+\gamma_{1}\right)^{2}\left(1+Y \beta_{1}+\gamma_{1}\right)^{2}}=0
\end{aligned}
$$

where both polynomials $P_{2}$ and $Q_{2}$ are of degree two. From the equation $Q_{2}(y, Y)=0$, we get $Y$ as a function of $y$, that is, $Y=f(y)$, and after substituting this expression in the equation $P_{2}(y, Y)=0$, we obtain a quadratic polynomial equation in the variable $y$. Then the maximum number of solutions of (33) is two, namely $\left(y_{1}, Y_{1}\right)$ and $\left(y_{2}, Y_{2}\right)$, but in fact, these two solutions represent the same limit cycle because they are symmetric as usual. Thus system (4)-(4) has at most one limit cycle. 
Now we give an example of a discontinuous piecewise differential system of type (4)(4) having one limit cycle. On $x<0$ we consider the quadratic isochronous differential system (23) and on $x>0$ we take the quadratic isochronous differential system of type (4)

$$
\dot{x}=-2-2 y+x^{2}+x y, \quad \dot{y}=2+2 x+3 y+x y+y^{2},
$$

with a first integral

$$
H_{3}(x, y)=\frac{(1+y)^{2}+(1+x+y)^{2}}{(2+y)^{2}}
$$

The solution of (33) satisfying $y<Y$ is $(y, Y)=\left(-\frac{4}{3}, 0\right)$, which provides the limit cycle for the discontinuous differential piecewise system (23)-(34) shown in Figure 9.

\section{B. Proof of statement (b) of Theorem 3.}

We consider again the quadratic polynomial differential system (32) with first integral $\widetilde{H}_{3}(x, y)$ in the half-plane $x<0$ and for $x>0$ we take the isochronous differential system (5) whose first integral is $H_{4}(x, y)$.

If there exists a limit cycle of the discontinuous piecewise differential system (32)-(5), then it has two different intersection points $(0, y)$ and $(0, Y)$ with the line $x=0$, which satisfy the system

$$
\begin{aligned}
\widetilde{H}_{3}(0, y)-\widetilde{H}_{3}(0, Y) & =\frac{(Y-y) P_{2}(y, Y)}{\left.\left(1+y \beta_{1}+\gamma_{1}\right)^{2}\left(1+Y \beta_{1}+\gamma_{1}\right)^{2}\right)}=0 \\
H_{4}(0, y)-H_{4}(0, Y) & =\frac{(Y-y) Q_{4}(y, Y)}{(-3+16 y \beta+16 \gamma)(-3+16 Y \beta+16 \gamma)}=0
\end{aligned}
$$

where the polynomials $P_{2}$ and $Q_{4}$ have degree two and four, respectively. From the equation $P_{2}(y, Y)=0$, we get $Y$ as a function of $y$, that is, $Y=f(y)$, and substituting this expression in the equation $Q_{4}(y, Y)=0$, we obtain a polynomial equation of degree six in the variable $y$. Then, the maximum number of solutions of (35) is six. But due to the symmetry of the solutions, the system (4)-(5) has at most three limit cycles.

Now, we show an example of discontinuous piecewise differential system of type (4)(5) having two limit cycles. On $x<0$ we consider the quadratic isochronous differential 
system

$$
\begin{aligned}
& \dot{x}=-5.92592-47.9127 x-58.6606 y+x^{2}-0.101841 x y, \\
& \dot{y}=-246.99+9.5224 x-26.911 y+x y-0.101841 y^{2},
\end{aligned}
$$

with the first integral

$$
\widetilde{H}_{3}(x, y)=\frac{(24.9412-x+0.101841 y)^{2}+(602.838-x+61 y)^{2}}{(603.838-x+61 y)^{2}}
$$

and on $x>0$ we consider the quadratic isochronous differential system (26) of type (5) whose first integral is 27 . The solutions of (35) satisfying $y<Y$ which provide the two limit cycles for the discontinuous differential piecewise system (36)-(26) are

$$
\left(y_{1}, Y_{1}\right)=(-1,1), \quad\left(y_{2}, Y_{2}\right)=(-2,3)
$$

shown in Figure 10.

\section{Proof of statement (c) of Theorem 3.}

We take the quadratic polynomial differential system (32) with the first integral $\widetilde{H}_{3}(x, y)$ in the half-plane $x<0$ and for $x>0$ we take the isochronous differential system (6) with the first integral $H_{5}(x, y)$.

If there exists a limit cycle of the discontinuous piecewise differential system (32)-(6), then it has two different intersection points $(0, y)$ and $(0, Y)$ with the separation line $x=0$, satisfying the system

$$
\begin{aligned}
& \widetilde{H}_{3}(0, y)-\widetilde{H}_{3}(0, Y)=\frac{(Y-y) P_{2}(y, Y)}{\left.\left(1+y \beta_{1}+\gamma_{1}\right)^{2}\left(1+Y \beta_{1}+\gamma_{1}\right)^{2}\right)}=0, \\
& H_{5}(0, y)-H_{5}(0, Y)=\frac{(Y-y) Q_{5}(y, Y)}{(3+8 y \beta+8 \gamma)^{4}(3+8 Y \beta+8 \gamma)^{4}}=0
\end{aligned}
$$

where the polynomials $P_{2}$ and $Q_{5}$ are of degree two and five, respectively. From the equation $P_{2}(y, Y)=0$, we obtain $Y$ as a function of $y$, that is, $Y=f(y)$, and putting this expression in the second equation $Q_{5}(y, Y)=0$, we obtain a polynomial equation of degree six in the variable $y$. Then, the maximum number of solutions of (37) is six, but 
due to the symmetry, there are at most three solutions of system (37) satisfying $y<Y$. Thus, system (4)-(6) has at most three limit cycles.

Next, we give an example of discontinuous piecewise differential system of type (4)(6) having two limit cycles. On $x<0$ we consider the quadratic isochronous differential system

$$
\begin{aligned}
& \dot{x}=6781.71+363.957 x-15585.4 y+x^{2}-45.2674 x y, \\
& \dot{y}=140.782+7.24945 x-308.767 y+x y-45.2674 y^{2},
\end{aligned}
$$

with the first integral

$$
\widetilde{H}_{3}(x, y)=\frac{(-70.4328+x-57.2448 y)^{2}+(18.3967+x-45.2674 y)^{2}}{(-69.4328+x-57.2448 y)^{2}}
$$

and on $x>0$ we consider the quadratic isochronous differential system (30) of type (6) whose first integral is 31 . The solutions of (37) satisfying $y<Y$ which provide the two limit cycles for the discontinuous differential piecewise system (38)-(30) are

$$
\left(y_{1}, Y_{1}\right)=(0.1,1), \quad\left(y_{2}, Y_{2}\right)=(0.166666,0.833335)
$$

shown in Figure 11.

\section{PROOF OF THEOREM 4}

\section{A. Proof of Theorem 4 for system (IV)-(IV)}

We consider the quadratic polynomial differential system (5) with the first integral $H_{4}(x, y)$ in the half-plane $x<0$. By changing the parameters $(a, \alpha, b, \beta, c, \gamma)$ to $\left(a_{1}, \alpha_{1}, b_{1}, \beta_{1}, c_{1}, \gamma_{1}\right)$ in system (5) and its first integral, we obtain a second isochronous 
quadratic differential system of type (5) with the first integral $\widetilde{H}_{4}(x, y)$, namely

$$
\begin{aligned}
& \dot{x}=\frac{1}{3 a_{1} \beta_{1}-3 b_{1} \alpha_{1}}\left(b_{1}\left(c_{1}+a_{1} x\right)\left(-3+16 x \alpha_{1}+8 y \beta_{1}+16 \gamma_{1}\right)+b_{1}^{2} y\left(-3+16 x \alpha_{1}+12 y \beta_{1}+16 \gamma_{1}\right)\right. \\
& -\beta_{1}\left(4 c_{1}^{2}+8 a_{1} c_{1} x+4 a_{1}^{2} x^{2}+3\left(x \alpha_{1}+y \beta_{1}+\gamma_{1}\right)\right) \\
& \dot{y}=\frac{1}{3 b_{1} \alpha_{1}-3 a_{1} \beta_{1}} a_{1}\left(c_{1}+b_{1} y\right)\left(-3+8 x \alpha_{1}+16 y \beta_{1}+16 \gamma_{1}\right)+a_{1}^{2} x\left(-3+12 x \alpha_{1}+16 y \beta_{1}+16 \gamma_{1}\right) \\
& -\alpha_{1}\left(4 c_{1}^{2}+8 b_{1} c_{1} y+4 b_{1}^{2} y^{2}+3\left(x \alpha_{1}+y \beta_{1}+\gamma_{1}\right)\right),
\end{aligned}
$$

whose first integral is

$$
\begin{aligned}
\widetilde{H}_{4}(x, y)= & \frac{1}{16\left(\gamma_{1}+\alpha_{1} x+\beta_{1} y\right)-3}\left(-24\left(a_{1} x+b_{1} y+c_{1}\right)^{2}\left(\gamma_{1}+\alpha_{1} x+\beta_{1} y\right)\right. \\
& \left.+9\left(\left(a_{1} x+b_{1} y+c_{1}\right)^{2}+\left(\gamma_{1}+\alpha_{1} x+\beta_{1} y\right)^{2}\right)+16\left(a_{1} x+b_{1} y+c_{1}\right)^{4}\right)
\end{aligned}
$$

If there exists a limit cycle of the discontinuous piecewise differential system (5)-(39), then it has two different intersection points $(0, y)$ and $(0, Y)$ with the separation line $x=0$, satisfying the system

$$
\begin{aligned}
\widetilde{H}_{4}(0, y)-\widetilde{H}_{4}(0, Y) & =\frac{(Y-y) P_{4}(y, Y)}{\left(-3+16 y \beta_{1}+16 \gamma_{1}\right)\left(-3+16 Y \beta_{1}+16 \gamma_{1}\right)}=0 \\
H_{4}(0, y)-H_{4}(0, Y) & =\frac{(Y-y) Q_{4}(y, Y)}{(-3+16 y \beta+16 \gamma)(-3+16 Y \beta+16 \gamma)}=0
\end{aligned}
$$

where both polynomials $P_{4}$ and $Q_{4}$ are of degree four. From the equation $P_{4}(y, Y)=0$, we obtain $Y$ as a function of $y$, that is, $Y=f(y)$, and substituting it in the second equation $Q_{4}(y, Y)=0$, we obtain a polynomial equation of degree six in the variable $y$. Then, the maximum number of solutions of (40) is six, but due to the symmetry, there are at most three solutions of system (40) satisfying $y<Y$. Thus, system (5)-(5) has at most three crossing limit cycles.

Next, we give an example of discontinuous piecewise differential system of type (5)(5) having two limit cycles. On $x<0$ we consider the quadratic isochronous differential 
system

$$
\begin{aligned}
& \dot{x}=-0.593985+6.89377 x-6.77032 y+3.03414 x^{2}-0.667964 x y-1.89916 y^{2}, \\
& \dot{y}=-4.77286+7.53417 x-9.75481 y+1.87138 x^{2}+1.93171 x y-3.30143 y^{2},
\end{aligned}
$$

with the first integral

$$
\begin{aligned}
\widetilde{H}_{4}(x, y)= & \frac{1}{-3+16(3.57331+x+1.22861 y)}\left(16(-0.35763+x-0.908852 y)^{4}\right. \\
& -24(-0.35763+x-0.908852 y)^{2}(3.57331+x+1.22861 y) \\
& \left.+9\left((-0.35763+x-0.908852 y)^{2}+(3.57331+x+1.22861 y)^{2}\right)\right),
\end{aligned}
$$

and on $x>0$ we consider the quadratic isochronous differential system

$$
\begin{aligned}
& \dot{x}=-4.35274+17.666 x-44.1167 y+1.65212 x^{2}-2.11558 x y-4.00174 y^{2}, \\
& \dot{y}=279.554+16.3982 x+89.6236 y-0.305758 x^{2}+4.69575 x y+5.2283 y^{2},
\end{aligned}
$$

with the first integral

$$
\begin{aligned}
H_{4}(x, y)= & \frac{1}{-3+16(47.304-x+12.0396 y)}\left(16(-13.4112-x-1.04263 y)^{4}\right. \\
& -24(-13.4112-x-1.04263 y)^{2}(47.304-x+12.0396 y) \\
& \left.+9\left((-13.4112-x-1.04263 y)^{2}+(47.304-x+12.0396 y)^{2}\right)\right) .
\end{aligned}
$$

The solutions of (40) satisfying $y<Y$ which provide the two limit cycles for the discontinuous differential piecewise system (41)-(43) are

$$
\left(y_{1}, Y_{1}\right)=(-1,1), \quad\left(y_{2}, Y_{2}\right)=(-2,3)
$$

shown in Figure 12.

\section{B. Proof Theorem 4 for system (IV)-(V)}

We take the quadratic polynomial differential system (39) with the first integral $\widetilde{H}_{4}(x, y)$ in the half-plane $x<0$ and for $x>0$ we take the isochronous differential 
system (6) with the first integral $H_{5}(x, y)$.

If there exists a limit cycle of the discontinuous piecewise differential system (39)-(6), then there exist two different intersection points $(0, y)$ and $(0, Y)$ with the separation line $x=0$, satisfying the system

$$
\begin{aligned}
\widetilde{H}_{4}(0, y)-\widetilde{H}_{4}(0, Y) & =\frac{(Y-y) P_{4}(y, Y)}{\left(-3+16 y \beta_{1}+16 \gamma_{1}\right)\left(-3+16 Y \beta_{1}+16 \gamma_{1}\right)}=0 \\
H_{5}(0, y)-H_{5}(0, Y) & =\frac{(Y-y) Q_{5}(y, Y)}{(3+8 y \beta+8 \gamma)^{4}(3+8 Y \beta+8 \gamma)^{4}}=0
\end{aligned}
$$

where the polynomials $P_{4}$ and $Q_{5}$ are of degree four and five, respectively. Again, we obtain $Y$ as a function of $y$, that is, $Y=f(y)$, from the equation $P_{4}(y, Y)=0$, and putting this expression in the second equation $Q_{5}(y, Y)=0$, we obtain a polynomial equation of degree seven in the variable $y$. Then, the maximum number of solutions of (44) is seven, but due to the symmetry, there are at most three solutions of system (44) which satisfy the condition $y<Y$. Then, system (5)-(6) has at most three limit cycles.

Now, we show an example of discontinuous piecewise differential system of type (5)-(6) having two limit cycles. On $x<0$ we consider again the quadratic isochronous differential system (41) whose first integral is (42), and on $x>0$ we consider the quadratic isochronous differential system

$$
\begin{aligned}
& \dot{x}=-0.0135199+6.44812 x-0.134872 y+4.00057 x^{2}+0.155285 x y-0.00453093 y^{2}, \\
& \dot{y}=15.1375+132.575 x-1.28187 y+22.8872 x^{2}+5.3322 x y-0.0778083 y^{2},
\end{aligned}
$$

with the first integral

$$
\begin{aligned}
H_{5}(x, y)= & \frac{1}{(3+8(-1.24193+x-0.0582816 y))^{4}}\left(9 \left((-1.24193+x-0.0582816 y)^{2}\right.\right. \\
& \left.+(0.387469+x-0.0000248632 y)^{2}\right)+24(-1.24193+x-0.0582816 y)^{3} \\
& \left.+16(-1.24193+x-0.0582816 y)^{4}\right)
\end{aligned}
$$

The solutions of (44) satisfying $y<Y$ which provide the two limit cycles for the discon- 
tinuous differential piecewise system (41)-(45) are

$$
\left(y_{1}, Y_{1}\right)=(-1,1), \quad\left(y_{2}, Y_{2}\right)=(-2,3)
$$

shown in Figure 13.

\section{PROOF OF THEOREM 5}

We consider the quadratic polynomial differential system (6) with the first integral $H_{5}(x, y)$ in the half-plane $x<0$. If we change the parameters $(a, \alpha, b, \beta, c, \gamma)$ to $\left(a_{1}, \alpha_{1}, b_{1}, \beta_{1}, c_{1}, \gamma_{1}\right)$ in system (6) and its first integral, we obtain a second isochronous quadratic differential system of type (6) with the first integral $\widetilde{H}_{5}(x, y)$, namely

$$
\begin{aligned}
\dot{x}= & \frac{1}{3\left(\alpha_{1} b_{1}-a_{1} \beta_{1}\right)}\left(4 \beta_{1} \gamma_{1}^{2}+8 b_{1} \gamma_{1} c_{1}+3 b_{1} c_{1}+3 \beta_{1} \gamma_{1}-16 \beta_{1} c_{1}^{2}+\left(8 a_{1} b_{1} \gamma_{1}+8 \alpha_{1} \beta_{1} \gamma_{1}\right.\right. \\
& \left.+3 a_{1} b_{1}+3 \alpha_{1} \beta_{1}-32 a_{1} \beta_{1} c_{1}+8 \alpha_{1} b_{1} c_{1}\right) x+\left(8 b_{1}^{2} \gamma_{1}+8 \beta_{1}^{2} \gamma_{1}+3 b_{1}^{2}+3 \beta_{1}^{2}\right. \\
& \left.-24 \beta_{1} b_{1} c_{1}\right) y+4\left(\alpha_{1}^{2} \beta_{1}-4 a_{1}^{2} \beta_{1}+2 \alpha_{1} a_{1} b_{1}\right) x^{2}+8\left(\alpha_{1} \beta_{1}^{2}-3 a_{1} \beta_{1} b_{1}+\alpha_{1} b_{1}^{2}\right) x y \\
& \left.-4 \beta_{1} y^{2}\left(2 b_{1}^{2}-\beta_{1}^{2}\right) y^{2}\right) \\
\dot{y}= & \frac{1}{3\left(\alpha_{1} b_{1}-a_{1} \beta_{1}\right)}\left(16 \alpha_{1} c_{1}^{2}-4 \alpha_{1} \gamma_{1}^{2}-8 a_{1} \gamma_{1} c_{1}-3 a_{1} c_{1}-3 \alpha_{1} \gamma_{1}-\left(8 a_{1}^{2} \gamma_{1}+8 \alpha_{1}^{2} \gamma_{1}\right.\right. \\
& \left.+3 a_{1}^{2}+3 \alpha_{1}^{2}-24 \alpha_{1} a_{1} c_{1}\right) x-\left(8 a_{1} b_{1} \gamma_{1}+8 \alpha_{1} \beta_{1} \gamma_{1}+3 a_{1} b_{1}+3 \alpha_{1} \beta_{1}+8 a_{1} \beta_{1} c_{1}\right. \\
& \left.-32 \alpha_{1} b_{1} c_{1}\right) y+4 \alpha_{1}\left(2 a_{1}^{2}-\alpha_{1}^{2}\right) x^{2}+8\left(a_{1}^{2}\left(-\beta_{1}\right)-\alpha_{1}^{2} \beta_{1}+3 \alpha_{1} a_{1} b_{1}\right) x y \\
& \left.-4\left(\alpha_{1} \beta_{1}^{2}+2 a_{1} \beta_{1} b_{1}-4 \alpha_{1} b_{1}^{2}\right) y^{2}\right)
\end{aligned}
$$

whose first integral is

$$
\begin{aligned}
H_{5}(x, y)= & \frac{1}{\left(8\left(\gamma_{1}+\alpha_{1} x+\beta_{1} y\right)+3\right)^{4}}\left(9\left(\left(a_{1} x+b_{1} y+c_{1}\right)^{2}+\left(\gamma_{1}+\alpha_{1} x+\beta_{1} y\right)^{2}\right)\right. \\
& \left.+16\left(\gamma_{1}+\alpha_{1} x+\beta_{1} y\right)^{4}+24\left(\gamma_{1}+\alpha_{1} x+\beta_{1} y\right)^{3}\right) .
\end{aligned}
$$

If there exists a limit cycle of the discontinuous piecewise differential system (6)-(46), then it has two different intersection points $(0, y)$ and $(0, Y)$ with the separation line 
$x=0$, satisfying the system

$$
\begin{aligned}
\widetilde{H}_{5}(0, y)-\widetilde{H}_{5}(0, Y) & =\frac{(Y-y) P_{5}(y, Y)}{\left(3+8 y \beta_{1}+8 \gamma_{1}\right)^{4}\left(3+8 Y \beta_{1}+8 \gamma_{1}\right)^{4}}=0, \\
H_{5}(0, y)-H_{5}(0, Y) & =\frac{(Y-y) Q_{5}(y, Y)}{(3+8 y \beta+8 \gamma)^{4}(3+8 Y \beta+8 \gamma)^{4}}=0,
\end{aligned}
$$

where both polynomials $P_{5}$ and $Q_{5}$ are of degree five. Then, by Bézout Theorem, the maximum number of solutions $(y, Y) \in \mathbb{C}$ of equation (47) is 25. Since our solutions appear in pairs because of the symmetry, the maximum number of solutions satisfying $y<Y$ is twelve.

Next, we give an example of discontinuous piecewise differential system of type (6)(46) having two limit cycles.

On $x<0$ we consider the quadratic isochronous differential system (48)

$$
\begin{aligned}
& \dot{x}=0.103814+0.0255717 x-0.0978649 y-0.0368897 x^{2}+0.218646 x y-0.322863 y^{2}, \\
& \dot{y}=0.03051+0.0170376 x-0.0573298 y-0.0153809 x^{2}+0.0905173 x y-0.132811 y^{2},
\end{aligned}
$$

with the first integral

$$
\begin{aligned}
\widetilde{H}_{5}(x, y)= & \frac{1}{(3+8(-4.15332+x-2.61606 y))^{4}}\left(9 \left((-3.79477+2 x-5.613 y)^{2}\right.\right. \\
& \left.+(-4.15332+x-2.61606 y)^{2}\right)+24(-4.15332+x-2.61606 y)^{3} \\
& \left.+16(-4.15332+x-2.61606 y)^{4}\right)
\end{aligned}
$$

and on $x>0$ we consider the quadratic isochronous differential system

$$
\begin{aligned}
\dot{x}= & 0.0000696792+0.00114119 x-0.000158087 y+0.000767513 x^{2}-0.0000760837 x y \\
& -0.0000043242 y^{2}, \\
\dot{y}= & 0.000494721+0.00630472 x-0.000840437 y+0.00219727 x^{2}+0.000153785 x y \\
& -0.0000622098 y^{2},
\end{aligned}
$$


with the first integral

$$
\begin{aligned}
H_{5}(x, y)= & \frac{1}{(3+8(-0.825206+x-0.195584 y))^{4}}\left(9 \left((-0.825206+x-0.195584 y)^{2}\right.\right. \\
& \left.+(0.178088+x-0.118725 y)^{2}\right)+24(-0.825206+x-0.195584 y)^{3} \\
& \left.+16(-0.825206+x-0.195584 y)^{4}\right)
\end{aligned}
$$

The solutions of (47) satisfying $y<Y$ which provide the two limit cycles for the discontinuous differential piecewise system (48)-(49) are

$$
\left(y_{1}, Y_{1}\right)=(0.1,1), \quad\left(y_{2}, Y_{2}\right)=(0.166666,0.833335)
$$

shown in Figure 14.

Remark. Equations (47) can have at most 12 solutions using Bézout Theorem, but numerical evidences obtained given arbitrary values to the parameters of the class of discontinuous piecewise differential systems of type $(V)-(V)$, we could only find at most 8 real solutions, but these solutions in general do not provide crossing limit cycles. In all the particular piecewise differential systems studied numerically we could only find at most 2 crossing limit cycles.

\section{CONCLUSIONS}

There is a unique family of linear isochronous centers and four families of quadratic isochronous centers. Considering all the possibilities of choosing two pairs of these isochronous centers, eventually repeated, we obtain fifteen pairs. Therefore we have fifteen classes of discontinuous piecewise differential systems formed by two differential systems separated by a straight line when these two differential systems are linear isochronous centers or quadratic isochronous centers.

For these fifteen classes of discontinuous piecewise differential systems we provide an upper bound for the maximum number of limit cycles that they can exhibit, i.e. for these classes of differential systems we have solved the 16th Hilbert problem. Moreover for seven of these fifteen classes of discontinuous piecewise differential systems the upper 
bound on the maximum number obtained is reached.

More precisely, it was known that discontinuous piecewise differential systems formed by two linear isochronous centers separated by a straight line cannot have limit cycles, $\mathrm{see}^{27}$. If one of the systems is a linear isochronous center and the other is a quadratic isochronous center then their maximum number of limit cycles is studied in Theorem 1 . While if the two systems are quadratic isochronous centers then their maximum number of limit cycles are studied in Theorems 2, 3, 4 and 5 .

\section{ACKNOWLEDGMENTS}

The first author is partially supported by the Ministerio de Economía y Competitividad in the frame of project PGC2018-096265-B-I00, and by the Consejería de Economía y Conocimiento de la Junta de Andalucía, under grant P12-FQM-1658. The second author is supported by the Ministerio de Ciencia, Innovación y Universidades, Agencia Estatal de Investigación grant PID2019-104658GB-I00 (FEDER), the Agència de Gestió d'Ajuts Universitaris i de Recerca grant 2017SGR1617, and the H2020 European Research Council grant MSCA-RISE-2017-777911. The third author is partially supported by by FCT/Portugal through UID/MAT/04459/2019.

\section{REFERENCES}

${ }^{1}$ J.C. Artés, J. Llibre, J.C. Medrado and M.A. Teixeira, Piecewise linear differential systems with two real saddles, Math. Comput. Simul., 95 (2013), 13-22.

${ }^{2}$ D.C. Braga And L.F. Mello, Limit cycles in a family of discontinuous piecewise linear differential systems with two zones in the plane, Nonlinear Dynam., 73 (2013), $1283-1288$.

${ }^{3}$ C. Buzzi, C. Pessoa And J. Torregrosa, Piecewise linear perturbations of a linear center, Discrete Contin. Dyn. Syst., 33 (2013), 3915-3936.

${ }^{4}$ J. Castillo, J. Llibre And F. Verduzco, The pseudo-Hopf bifurcation for planar discontinuous piecewise linear differential systems, Nonlinear Dynam., 90 (2017), $1829-1840$. 
${ }^{5}$ J. Chavarriga And M. Sabatini, A survey on isochronous centers, Qual. Th. Dyn. Syst, 1 (1999), 1-70.

${ }^{6}$ S. CoOmbes, Neuronal networks with gap junctions: a study of piecewise linear planar neuron models, SIAM J. Appl. Dyn. Syst., 7 (2008), 1101-1129.

${ }^{7}$ M. di Bernardo, C.J. Budd, A.R. Champneys and P. Kowalczyk, Piecewisesmooth dynamical systems: theory and applications, Applied Mathematical Sciences, Springer, 2008.

${ }^{8}$ R.D. EuzÉBIO AND J. LliBRE, On the number of limit cycles in discontinuous piecewise linear differential systems with two pieces separated by a straight line, J. Math. Anal. Appl., 424 (2015), 475-486.

${ }^{9}$ A.F. FilipPov, Differential equations with discontinuous right-hand sides, translated from Russian. Mathematics and its Applications (Soviet Series) vol. 18, Kluwer Academic Publishers Group, Dordrecht, 1988.

${ }^{10}$ E. Freire, E. Ponce, F. Rodrigo And F. Torres, Bifurcation sets of continuous piecewise linear systems with two zones, Int. J. Bifurcation and Chaos., 8 (1998), 2073-2097.

${ }^{11}$ E. Freire, E. Ponce And F. Torres, Canonical discontinuous planar piecewise linear systems, SIAM J. Applied Dynamical Systems., 11 (2012), 181-211.

${ }^{12}$ E. Freire, E. Ponce And F. TORREs, A general mechanism to generate three limit cycles in planar Filippov systems with two zones, Nonlinear Dynamics, 78 (2014), $251-263$.

${ }^{13} \mathrm{~F}$. Giannakopoulos And K. Pliete, Planar systems of piecewise linear differential equations with a line of discontinuity, Nonlinearity, 14 (2001), 1611-1632.

${ }^{14}$ M.R.A. Gouveia, J. Llibre And D D. Novaes, On limit cycles bifurcating from the infinity in discontinuous piecewise linear differential systems, Appl. Math. Comput., 271 (2015), 365-374.

${ }^{15}$ D. Hilbert, Mathematische Probleme, Lecture, Second Internat. Congr. Math. (Paris, 1900), Nachr. Ges. Wiss. G"ottingen Math. Phys. KL. (1900), 253-297; English transl., Bull. Amer. Math. Soc. 8 (1902), 437-479; Bull. (New Series) Amer. Math. Soc. 37 (2000), 407-436. 
${ }^{16}$ S.M. HUAN AND X.S. YANG, On the number of limit cycles in general planar piecewise systems, Discrete Cont. Dyn. Syst., Series A 32 (2012), 2147-2164.

${ }^{17}$ S.M. HUAN AND X.S. YANG, Existence of limit cycles in general planar piecewise linear systems of saddle-saddle dynamics, Nonlinear Anal., 92 (2013), 82-95.

${ }^{18}$ S.M. HUAN AND X.S. YANG, On the number of limit cycles in general planar piecewise linear systems of node-node types, J. Math. Anal. Appl., 411 (2014), 340-353.

${ }^{19}$ Yu. Ilyashenko, Centennial history of Hilbert's 16th problem, Bull. (New Series) Amer. Math. Soc. 39 (2002), 301-354.

${ }^{20} \mathrm{~J}$. LI, Hilbert's 16 th problem and bifurcations of planar polynomial vector fields, Internat. J. Bifur. Chaos Appl. Sci. Engrg. 13 (2003), 47-106.

${ }^{21} \mathrm{~L}$. LI, Three crossing limit cycles in planar piecewise linear systems with saddle-focus type, Electron. J. Qual. Theory Differ. Equ., 70 (2014), 14 pp.

${ }^{22}$ J. Llibre, D.D. Novaes And M.A. TeixeIRA, Limit cycles bifurcating from the periodic orbits of a discontinuous piecewise linear differential center with two zones, Int. J. Bifurcation and Chaos, 25 (2015), 1556144, 11 pp.

${ }^{23}$ J. Llibre, D.D. Novaes AND M.A. TeIXeIRA, Maximum number of limit cycles for certain piecewise linear dynamical systems, Nonlinear Dyn., 82 (2015), 1159-1175.

${ }^{24}$ J. Llibre, D.D. Novaes And M.A. Teixeira, On the birth of limit cycles for nonsmooth dynamical systems, Bull. Sci. Math., 139 (2015), 229-244.

${ }^{25}$ J. Llibre, M. OrdóÑEz ANd E. PONCE, On the existence and uniqueness of limit cycles in planar piecewise linear systems without symmetry, Nonlinear Anal. Series B: Real World Appl., 14 (2013), 2002-2012.

${ }^{26}$ J. LliBRE AND E. PONCE, Three nested limit cycles in discontinuous piecewise linear differential systems with two zones, Dyn. Cont. Disc. Impul. Syst., Series B, 19 (2012), $325-335$.

${ }^{27}$ J. Llibre And M.A. TeIXEIRA, Piecewise linear differential systems without equilibria produce limit cycles?, Nonlinear Dyn., 88 (2017), 157-164.

${ }^{28}$ J. Llibre, M.A. Teixeira And J. Torregrosa, Lower bounds for the maximum number of limit cycles of discontinuous piecewise linear differential systems with a straight line of separation, Int. J. Bifurcation and Chaos, 23 (2013), 1350066, 10 pp. 
${ }^{29}$ J. Llibre AND X. ZhANG, Limit cycles for discontinuous planar piecewise linear differential systems separated by one straight line and having a center, J. Math. Anal. Appl., 467 (2018), 537-549.

${ }^{30} \mathrm{R}$. Thul And S. CoOmBes, Understanding cardiac alternans: a piecewise linear modeling framework, Chaos. An Interdisciplinary Journal of Nonlinear Science, 20 (2010), 045102, 13 pp.

${ }^{31} \mathrm{~A}$. Tonnelier, McKean caricature of the FitzHugh-Nagumo model: traveling pulses in a discrete diffusive medium, Physical Review E. Statistical, Nonlinear, and Soft Matter Physics, 67 (2003), 036105, 9 pp.

${ }^{32}$ A. Tonnelier And W. Gerstner, Piecewise linear differential equations and integrate-and-fire neurons: insights from two-dimensional membrane models, Physical Review E. Statistical, Nonlinear, and Soft Matter Physics, 67 (2003), 021908, 16 pp.

${ }^{33}$ A. Visintin, Differential models of hysteresis, Applied Mathematical Sciences vol. 111, Springer-Verlag, Berlin 1994. 